\title{
Bilateral and Community Enforcement in a Networked Market with Simple Strategies*
}

\author{
Itay P. Fainmesser ${ }^{\dagger} \quad$ David A. Goldberg F $^{\ddagger}$
}

January 5, 2011

\begin{abstract}
We present a model of repeated games in large buyer-seller networks in the presence of reputation networks via which buyers share information about past transactions. The model allows us to characterize cooperation networks - networks in which each seller cooperates (by providing high quality goods) with every buyer that is connected to her. To this end, we provide conditions under which: [1] the incentives of a seller $s$ to cooperate depend only on her beliefs with respect to her local neighborhood - a subnetwork that includes seller $s$ and is of a size that is independent of the size of the entire network; and [2] the incentives of a seller $s$ to cooperate can be calculated as if the network was a random tree with seller $s$ at its root. Our characterization sheds light on the welfare costs of relying only on repeated interactions for sustaining cooperation, and on how to mitigate such costs.
\end{abstract}

Keywords: Networks, moral hazard, graph theory, repeated games.

\section{Introduction}

In many markets, successful execution of mutually beneficial economic transactions relies on informal contracts that are enforced by social pressure and reputation. ${ }^{1}$ Informal

\footnotetext{
${ }^{*}$ We are grateful to Susan Athey, Drew Fudenberg, and Al Roth, for many discussions and invaluable comments. We also benefited from the help of Ilan Lobel and Theo Weber, and from the useful comments of James Burns, Ben Golub, Roberto Serrano, Adrien Vigier, and seminar participants at Harvard University and Microsoft Research New England.

${ }^{\dagger}$ Corresponding author. Department of Economics, Brown University, Providence, RI 02906. E-mail: Itay_Fainmesser@Brown.edu

${ }^{\ddagger}$ Operations Research Center, MIT, Cambridge, MA, 02139, E-mail: dag3141@mit.edu

${ }^{1}$ Macaulay (1963) points out that social pressure and reputation are perhaps more widely used than formal contracts and enforcement.
} 
enforcement mechanisms include personal and community enforcement mechanisms. ${ }^{2}$ It is by now widely recognized that when transactions between two parties are sufficiently frequent, personal enforcement is highly effective. ${ }^{3}$ Community enforcement can overcome the limitations of personal enforcement when transactions between two parties are infrequent, yet transactions in the population are frequent. In large markets, community enforcement is effective if third-party observability is available. ${ }^{4}$

However, despite the abundance of research on repeated games and community enforcement, the frequency of interactions, as well as the level of third-party observability are mostly treated as 'black boxes' or modeled for highly specialized cases. For example, in much of the literature, either any two parties interact in every period or random matching is assumed. In contrast, it is well known that in many markets with buyers and sellers, each buyer has access to a different subset of the sellers in the market, and chooses to purchase from even a smaller subset. Moreover, each buyer often learns about the outcomes of a different subset of the interactions in the market. ${ }^{5}$

To address these issues, we develop a model of repeated interactions and community enforcement in networked markets with buyers and sellers. Consider a group of sellers with limited supply and a group of buyers with limited demand. Each buyer can purchase a good from only a subset of sellers to whom he is connected. The connections between buyers and sellers define a buyer-seller trade network $G$. In every period, sellers meet sequentially with buyers that are connected to them and decide whether to exert costly effort and provide a high quality product (cooperate) or to shirk (defect). If a buyer $b$ purchases a good from seller $s$, he learns the quality of the good (after the purchase). A subset of the other buyers also learn the quality of the good that $b$ purchased from $s$; such buyers are said to be connected to buyer $b$. The connections between buyers define a reputation network $R$ that captures the level of third-party observability in the

\footnotetext{
${ }^{2}$ In personal enforcement mechanisms cheating triggers retaliation by the victim, whereas in community enforcement mechanisms dishonest behavior against one partner causes sanctions by several members in the society.

${ }^{3}$ For a good survey on long term relationships see Mailath and Samuelson (2006).

${ }^{4}$ See Kandori (1992), Greif (1993), and Ellison (1994).

${ }^{5}$ The economic literature offers extensive evidence for the presence of networks of cooperation and trust within markets. For example, see Fafchamps (1996), McMillan and Woodruff (1999), Hardle and Kirman (1995), Kirman and Vriend (2000), Weisbuch et al. (1996), and Karlan et al. (2009).
} 
market. The combined network, $N=(G, R)$ captures the market structure. We then ask the following questions: what structures of the network $N$ allow for equilibria in which only high quality goods are traded? How does the answer depend on the patience of sellers? What is the optimal network structure that allows for the maximal volume of trade in high quality goods, and can we do better with formal contracts?

We provide conditions under which a network $N$ is a Cooperation Network - a network in which a seller that expects all other sellers to always cooperate finds it optimal to always cooperate (with buyers that are connected to her). As expected, we find that adding a large number of links to a reputation network $R$ increases the set of buyer-seller networks $G$ such that $N=(G, R)$ is a cooperation network (Proposition 1$)$. When $R$ is sufficiently dense we find that networks in which there are fewer sellers, each having more connections (in $G$ ), and more buyers, each having fewer connections (in $G$ ), are cooperation networks for a larger set of discount factors (Proposition 2). Finally, we show that a sufficiently dense reputation network $R$ guarantees that the fraction of trade lost due to the incentive constraints goes to zero as the size of the market grows to infinity (Corollary 2). This is in spite of the fact that the optimal cooperation network achieves only a fraction $(<1)$ of the volume of trade that formal contracts could facilitate in any finite market with significantly more sellers than buyers - a fact that is driven by the observation that in every cooperation network some sellers are permanently excluded from the market.

Much of the previous work on games in networks analyzes static network games (Galeotti et al. 2010, Ballester et at. 2006, Bramoullé, D'Amours, and Kranton 2010). In static network games a player's payoff depends only on the actions taken by her immediate neighbors. As a result, beliefs on the network structure are used by a player only to establish a prior over the actions that her neighbors will take, and Galeotti et al. (2010) find that assuming that a player has incomplete knowledge of the network structure simplifies the analysis. In contrast, in our framework, due to the dynamic nature of the interactions and the limited supply and demand, a seller's incentives to cooperate generally depend on the entire network structure and on the actions taken by all of the buyers and sellers in the network. In large markets this makes the problem 
prohibitively complex, and there is no a priori reason to expect that assuming incomplete knowledge of the network structure will simplify the problem. In fact, in any finite network, a seller who has incomplete knowledge of the network is required to compute her incentives in every network that has positive probability according to her prior.

Thus, our contributions in this paper are two-fold, making both a new methodological contribution in the form of a novel method for moving beyond the framework in which a player's payoff function depends only on the actions taken by her immediate neighbors, and a more applied contribution in the form of a study of repeated interactions and community enforcement in networked markets with buyers and sellers.

At the core of our methodological contribution is a new method for reducing questions about the global properties of a network (e.g. equilibrium in which payoffs depend on all other players in the network) to questions about the local properties of the network. This allows us to provide conditions under which the incentives of a seller $s$ to cooperate with buyer $b$ depend only on her beliefs with respect to her local neighborhood - a subnetwork that includes seller $s$ and is of a size that is independent of the size of the entire network (Theorem 1). Thus, we are able to analyze large networks as if they were small. To derive these 'local conditions' we make use of recent results in the graph theory literature by Gamarnik and Goldberg (2010) - hereby GG - who study a simple matching algorithm called GREEDY and ask the following question: "When does the performance of GREEDY depend only on the local properties of the network?" By relating our market dynamics to the dynamics of GREEDY, we are able to use the tools developed in GG to analyze market equilibria. We note that the question of when the global properties of a graph are determined by the graph's local structure (a.k.a. a decay of correlations phenomenon occurs) has a long history. ${ }^{6}$

Along the same lines, we propose a natural model of beliefs over the network structure that captures the idea that: [1] there is a strong random component in the formation of

\footnotetext{
${ }^{6}$ Lauer et al. (2007) and Goring et al. (2007) study localization phenomenon for greedy algorithms, and Bayati et al. (2007) study the correlation decay phenomenon for the matchings of a graph. We also note that several recent works study related questions pertaining to when an (approximate) Nash equilibrium can be computed in a distributed / local manner using the tools of correlation decay, see e.g. Weber (2010) and Kanoria et al. (2010).
} 
networks, and [2] each seller knows more about her immediate neighborhood than about the rest of the network. For this model of beliefs, we prove that if the network $N$ is large and all other sellers always cooperate, then the incentives of seller $s$ to cooperate in $N$ can be approximated by the incentives of $s$ to cooperate in a simple network - a random tree with known distributions over the numbers of connections of buyers and sellers in the network. The approximation improves as the network grows and the error goes asymptotically to zero (Theorem 2). This result is based on a key graph theoretic lemma that we prove: consider a large bipartite graph $G$ that is chosen uniformly at random (u.a.r.) conditional on the (finite support) distributions of the number of links attached to nodes in the graph, then $G$ is asymptotically locally like a random tree (Lemma 2). Although results of a similar flavor are well-known in the random-graph community (see Wormald 1999 and the references therein), they have not been used in the economics literature. We hope that this paper helps to bridge between the two.

We also note that Lemma 2 provides a microfoundation to previous reduced form assumptions used in the networks literature. For example, Jackson and Yariv (2007) assume that each player in a network has expectations on the number of connections of each of the other players connected to her that are captured by a fixed degree distribution. Our result provide sufficient conditions under which this assumption is consistent with a common prior. ${ }^{7}$

Networks have been used to model market structure in many recent works in economics (see Jackson 2008 and Goyal 2007 for extensive surveys). In particular, most of the literature on buyer-seller networks has focused on the way that the network structure interacts with the price setting procedure (see also Kranton and Minehart 2001, and Blume et. al. 2007).

This paper is more closely related to the very recent developments in the study of repeated games in networks (see Kinateder 2008, Lippert and Spagnolo 2006, Mihm et al. 2009, Jackson et al. 2010, and works in progress by Miller and Nageeb as well as by Nava

\footnotetext{
${ }^{7}$ Kets (2010) shows that the assumed independence in Jackson and Yariv (2007) does not allow them to explore the entire range of strategic outcomes. Lemma 2 provides sufficient conditions under which the results by Jackson and Yariv overcome Kets' critique.
} 
and Piccione) and to the literature on trust and social collateral (see Karlan et al. 2009). An important difference from this literature is that we separate the analysis of the trade network from that of the communication network and allow both to vary in economically meaningful ways. Moreover, in our model, buyers and sellers have capacity constraints. In particular, supply and demand do not grow automatically with the number of an agent's links. This setup is realistic in many markets in which the roles of buyers and sellers are well defined.

Finally, most related to this paper is Fainmesser (2010a) who uses a similar framework. Fainmesser (2010a) characterizes cooperation networks in markets in which thirdparty observability is nonexistent ( $R$ is the empty reputation network), such as in many developing countries or in markets in which strategic considerations prevent the diffusion of information. In such markets, incentive constraints restricts the volume of trade even in large markets. The framework presented here is more general and allows for different levels of third-party observability.

\subsection{Third-party observability and Word-Of-Mouth (WOM)}

The source and degree of third-party observability among traders in real-world markets varies widely across contexts. ${ }^{8}$ One especially natural interpretation of $R$ is as a network of WOM.

Studying the interplay between WOM, the structure of the trade network, and cooperation is especially interesting due to recent changes in technology that affect the extent and nature of WOM: traditionally, WOM disseminated in small reputation networks between friends, neighbors, and family members. The internet extended these networks; we read user reviews before we buy a computer or book a hotel, consult Yelp.com before going to a restaurant or choosing a mechanic, and look at a prominent economist's website to find recommendations for cafes around the world. ${ }^{9,10}$

There are many theories of different aspects of WOM in networks. Acemoglu et

\footnotetext{
${ }^{8}$ See also Esfahani and Salehi-Isfahani (1989) and Banerjee and Newman (1998).

${ }^{9}$ See http://arielrubinstein.tau.ac.il/univ-coffee.html

${ }^{10}$ The idea that consumers are affected by reported experience of other people is obvious in online communities such as Yelp.com and Chowhound.com. In the context of reputation systems, Resnick and Zeckhauser (2002) show that sellers with better reputations are more likely to sell their items.
} 
al. (2010), DeMarzo et al. (2003), Ellison and Fudenberg (1995), Gale and Kariv (2003), Golub and Jackson (2010), and Niehaus (2010) study information aggregation in networks, whereas Galeotti and Goyal (2009), and Niehaus (2010) consider the effect of WOM on the diffusion of new products. There is also an extensive literature on the incentives for truthful reporting of past transactions to centralized reputation systems and on the informativeness of reputation scores. ${ }^{11}$ In this paper, we suggest that WOM can be captured by a reputation network in which connected buyers are able to share information credibly with each other.

\subsection{Outline}

In section 2 we lay out our model of a networked market, discuss the knowledge of sellers with respect to the network structure, and define a notion of a cooperation network. We conclude the section by setting up the planner's problem and defining a notion of efficiency loss that captures the fraction of transactions that must be forfeited in order for cooperation to persist. In section 3 we review our main results. Section 4 reviews the implications of our results to the analysis of social networks and suggests that our results provide new insights that are useful for the study of market structure more broadly. In section 5 we offer concluding remarks.

\section{Model}

Consider a market with a set of sellers $S \equiv\left\{1,2, \ldots, n_{s}\right\}$ and a set of buyers $B \equiv$ $\left\{1,2, \ldots, n_{b}\right\}$. Time is discrete $(t=1,2, \ldots, \infty)$. Sellers live forever and have a discount factor $\delta$. In every period, each seller is active with probability $\mu \in[0,1]$ and inactive otherwise. The realization of whether a seller is active in a given period is i.i.d. across sellers and periods. In a period in which a seller $s$ is active, she can choose to produce at most one unit of exactly one of two goods, $H$ or $L$. Producing $H$ has a positive cost of $c>0$, whereas the production of $L$ is costless. We interpret $H$ and $L$ to be quality variants of the same good that are indistinguishable to the buyers before purchase.

\footnotetext{
${ }^{11}$ See Dellarocas (2000 and 2001), Bolton et al. (2009), Resnick et al. (2000), and Resnick and Zeckhauser (2002).
} 
An inactive seller cannot produce. The probability that a seller is active $(\mu)$ captures potential noise in the production technology. Introducing noise in quality determination of the product does not make any difference in the analysis as long as a seller knows the quality that she produces.

We abstract from the price determination mechanism and assume that the ongoing price for a good is $\pi{ }^{12}$ For price $\pi$, each buyer $b$ has demand for one unit of $H$ in every period. Buyers have no demand for $L$ (even for a price of zero).

Sellers and buyers are embedded in a two-sided network of connections between buyers and sellers. A buyer can purchase only from sellers that are connected to her. In every period, meetings between sellers and buyers that are connected in the network occur in a random order, i.i.d. across periods. Formally, the buyer-seller network is represented by a bipartite graph $G$, with buyer partite $B$ (one node for each of the $n_{b}$ buyers) and seller partite $S$ (one node for each of the $n_{s}$ sellers). Let $E \subseteq S \times B$ be the set of edges in the graph. An edge $(s, b)$ between seller $s$ and buyer $b$ represents a potential interaction. In each period $t$, edges are chosen in an order $\sigma^{t}$ selected uniformly at random (u.a.r.), independent of all else, from the set of all $|E|$ ! possible orderings on the edges of $G$. When an edge $(s, b)$ is chosen, the potential interaction between seller $s$ and buyer $b$ is realized. ${ }^{13}$

We now explain informally the decisions that seller $s$ and buyer $b$ make when the potential interaction between $s$ and $b$ is realized. The decision of buyer $b$ is of the form: 'conditional on me not having already bought a unit of good and seller $s$ being active and seller $s$ not having already sold a unit of any good in the current period, will I purchase a unit of the good from $s$ ?'. The decision of an active seller $s$ is of the form: 'conditional on me not having already produced and sold a unit of a good in the current period, and assuming buyer $b$ is willing to purchase from me, will I produce and sell $H$ to $b$ (cooperate), or $L$ to $b$ (deviate)?'. We assume that an active seller $s$ cannot refuse a trade with a willing buyer, and thus $s$ cannot choose to produce nothing for a

\footnotetext{
${ }^{12}$ Any reasonable bargaining model will preserve the main insights of this paper. Incidentally, in a bargaining procedure in which sellers make take-it-or-leave-it offers, it is straightforward to construct equilibria for which our analysis goes through without changes.

${ }^{13}$ Our analysis is independent of whether sellers and buyers learn $\sigma^{t}$ after it is chosen.
} 
buyer $b$ that is willing to purchase from $s$ (assuming $s$ has not yet made any sales that period). An inactive seller does not have any decision to make. Note that a seller $s$ does not produce a product during period $t$ until she encounters a buyer who is willing to purchase from $s$ during $t$ (e.g. seller $s$ is a service provider).

We now formalize the above. For a given seller $s$ (buyer $b$ ), let $N_{1}(s) \equiv\{b \in B \mid(s, b) \in E\}$ $\left(N_{1}(b) \equiv\{s \in S \mid(s, b) \in E\}\right)$ denote the set of buyers (sellers) with which $s(b)$ may potentially interact. At the start of each period $t$, each buyer $b$ constructs a trust function $J_{b}^{t}: N_{1}(b) \rightarrow\{0,1\}$, where $J_{b}^{t}(s)=1(0)$ implies that conditional on $b$ not having already bought a unit of good and seller $s$ not having already sold a unit of a good by the time potential interaction $(s, b)$ is realized in $\sigma^{t}$, buyer $b$ will (won't) be willing to purchase a unit of a good from $s$. Similarly, at the start of each period $t$ each active seller $s$ constructs a quality function $Q_{s}^{t}: N_{1}(s) \rightarrow\{0,1\}$, where $Q_{s}^{t}(b)=1(0)$ implies that conditional on $s$ not having already produced and sold a unit of a good and buyer $b$ not having already purchased a unit of good by the time potential interaction $(s, b)$ is realized in $\sigma^{t}$ and $J_{b}^{t}(s)=1, s$ will produce and sell a unit of $H(L)$ to $b$.

\section{$2.1 \quad$ Reputation networks}

Each buyer has access to information about the outcomes of all of his past transactions, as well as limited information about other buyers' past transactions which he learns through his reputation network (e.g. via Word-Of-Mouth, reputation systems, or other mechanisms for third-party observability). More formally, there is a (reputation) graph $R$ on node set $B$, where edge $\left(b, b^{\prime}\right)$ is present in $R$ if and only if $b$ is informed when any seller $s$ sells either $H$ or $L$ to buyer $b^{\prime}$. The self-loop $(b, b)$ is present in $R$ for all $b$, and the graph is undirected (and thus $\left(b, b^{\prime}\right) \in R$ if and only if $\left(b^{\prime}, b\right) \in R$ ). Our results extend immediately to the directed graph case as well. Let $R\left(b, b^{\prime}\right)$ denote the indicator for whether or not $\left(b, b^{\prime}\right) \in R$. We also let $N_{R}(b) \triangleq \bigcup_{b^{\prime}: R\left(b, b^{\prime}\right)=1} b^{\prime}$ denote the set of buyers that share with buyer $b$ observations of past transactions, and $E_{R}^{s}(b) \triangleq$ $\bigcup_{b^{\prime} \in N_{R}(b) \cap N_{1}(s)}\left(s, b^{\prime}\right)$ denote the set of edges (in $G$ ) between seller $s$ and buyers that share with buyer $b$ information about past transactions.

We are interested in the incentives of sellers to produce $H$ conditional on the structure 
of $G$ and $R$. To this end, we simplify the analysis by restricting attention to buyers that use the following heuristic: each buyer agrees to purchase from exactly those sellers who (to the best of his knowledge) have never deviated and sold anyone a good $L$. We call this heuristic a reputation based grim trigger strategy and analyze below Bayesian equilibria of the sellers' behaviors given this buyers' strategy. Fainmesser (2010a) verifies that the buyers' strategies considered here are equilibrium strategies in the entire family of equilibria considered.

Formally, for each seller $s$ and buyer $b$, let $I^{t}(s, b)$ denote the indicator of the event that $s$ sold a unit of a good to $b$ in period $t$. Then at period 1 , each buyer $b$ sets $J_{b}^{1}(s)=1$ for all $s \in N_{1}(b)$ - that is, each buyer begins with the assumption that all sellers that are connected to him are honest and agrees to purchase from any of them. At the start of period $t$ (for $t \geq 2$ ), buyer $b$ sets

$$
J_{b}^{t}(s)=\max \left(J_{b}^{t-1}(s)-\sum_{b^{\prime} \in B} R\left(b, b^{\prime}\right) I^{t-1}\left(s, b^{\prime}\right)\left(1-Q_{s}^{t-1}\left(b^{\prime}\right)\right), 0\right) .
$$

Given $\left\{I^{t}(s, b)\right\}_{t=1}^{\infty}$ and $\left\{Q_{s}^{t}\right\}_{t=1}^{\infty}$, the total payoff of seller $s$ is

$$
u_{s}=\sum_{t=1}^{\infty} \delta^{t-1} \sum_{b \in N(s)} I^{t}(s, b)\left(\pi-c \cdot Q_{s}^{t}(b)\right)
$$

We notice that $I^{t}(s, b)$ depends on $N, \sigma^{t}$, and $\left\{Q_{s^{\prime}}^{\tau}(b)\right\}_{s^{\prime} \in S, \tau=1, \ldots, t}$. Consequently, the sellers' information and beliefs with respect to the network structure $N$ play a major role in sellers' expectations over their utility from choosing strategies $\left\{Q_{s}^{t}\right\}_{t=1}^{\infty}$.

\subsection{The network structure - sellers' knowledge and beliefs}

As we will primarily be concerned with the case in which the network is very large, it would be unreasonable to assume that each seller has complete knowledge of $N$. We first describe a general framework that captures any sellers' beliefs about the network structure and then suggest one particularly natural model in which sellers have incomplete knowledge of the network structure and stochastic beliefs with respect to their missing information.

Consider a seller $s$ that assigns some probability distribution $\mathcal{D}_{s}$ on the set of all 
possible networks $\mathcal{N}$, where a member of the set $\mathcal{N}$ is specified by a 2 -vector $N=(G, R)$ consisting of both a buyer-seller graph $G$ and a reputation graph $R$. We call $\mathcal{D}_{s}$ a belief of seller $s .^{14}$

Note that a seller's global beliefs - her beliefs about the global structure of the network - may be complicated, while her local beliefs may be more tractable. By global beliefs, we will generally be referring to her beliefs about the potential interactions between buyers and sellers separated from her in the network by a distance on the same order as the entire network. By local beliefs, we will generally be referring to her beliefs about the potential interactions between buyers and sellers separated from her in the network by a distance that is some small constant (e.g. 20) whose order is much smaller than that of the entire network, which may be arbitrarily large.

To explain the idea of local versus global beliefs more formally we need to define the notion of a distance between two nodes in a graph. A path of length $l$ in $G$ between node $v$ and node $v^{\prime}$ is a sequence of edges $\left\{\left(v_{0}, v_{1}\right),\left(v_{1}, v_{2}\right), \ldots,\left(v_{l-1}, v_{l}\right)\right\}$ such that $v_{0}=v$, $v_{l}=v^{\prime}$, and for every $i \in\{1,2, . ., l\},\left(v_{i-1}, v_{i}\right) \in G$. We say that the distance between $v$ and $v^{\prime}$ in $G$ is $l$ if the length of the shortest path between node $v$ and node $v^{\prime}$ in $G$ equals $l$.

We now make the distinction between local and global beliefs more formal. For a given seller $s$, recall that $N_{1}(s)$ is the set of buyers adjacent to $s$, and let $N_{2}(s)$ denote the set that includes the set of buyers in $N_{1}(s)$ as well as the set sellers adjacent to the buyers in $N_{1}(s)$, etc. In other words, a node $v$ is in $N_{d}(s)$ if and only if the length of the shortest path in $G$ between $s$ and $v$ does not exceed $d$. For a given belief distribution $\mathcal{D}_{s}$, we let $\mathcal{D}_{s}^{d}$ denote the distribution induced by $\mathcal{D}_{s}$ on $N_{d}(s)$. For example, if $\mathcal{D}_{s}$ places probability 1 on the complete binary tree with 100 levels and $s$ at the root, then $\mathcal{D}_{s}^{3}$ would place probability 1 on the complete binary tree with 3 levels and $s$ at the root.

To measure how different two belief distributions are we use the notion of total variational distance. For two random variables (r.v.) $X, Y$ with support on some countably infinite set $\mathcal{X}$, the total variational distance between $X$ and $Y, T V D(X, Y)$, is

\footnotetext{
${ }^{14}$ By definition, any network formation process, either deterministic or stochastic, induces a probability distribution over the set of all possible networks, and can be captured by some $\left\{\mathcal{D}_{s}\right\}_{s \in S}$.
} 
defined as $\sum_{x \in \mathcal{X}}|\operatorname{Pr}(X=x)-\operatorname{Pr}(Y=x)|$. Then for two belief distributions $\mathcal{D}_{s}, \mathcal{D}_{s^{\prime}}$, $T V D\left(\mathcal{D}_{s}^{d}, \mathcal{D}_{s}^{\prime d}\right)<\epsilon$ implies that the belief seller $s$ has about her depth-d neighborhood under $\mathcal{D}_{s}$ is 'within $\epsilon$ ' of the belief seller $s$ has about her depth- $d$ neighborhood under $\mathcal{D}_{s}^{\prime}$.

For a given network $N=(G, R)$ and a node $v \in S \cup B$, let $d_{v} \equiv\left|N_{1}(v)\right|$ denote the degree (number of neighbors) of $v$ in $G$, and let $\operatorname{deg}(N)$ denote the supremum, taken over all buyers and sellers $v \in S \cup B$, of $d_{v}$. For a given belief $\mathcal{D}_{s}$, let $\operatorname{deg}\left(\mathcal{D}_{s}\right)$ denote the supremum, over all networks $N$ to which $\mathcal{D}_{s}$ assigns strictly positive probability, of $\operatorname{deg}(N)$. Note that $\operatorname{deg}\left(\mathcal{D}_{s}\right)$ may be infinite.

Finally, for simplicity, we assume throughout that sellers do not use information from their interactions throughout the game to update their beliefs on the network structure $\left(\left\{\mathcal{D}_{s}\right\}_{s \in S}\right)$. While clearly restrictive, we believe that the analysis of repeated games with fixed beliefs on the network structure is an important first step and that our results are qualitatively robust. The analysis of learning the network structure through repeated interactions is beyond the scope of this paper and is left for future research. In addition, Fainmesser (2010a) proposes an example of a network generating process in which small changes to the network structure prevent sellers from learning the exact network structure beyond certain comparative statics. A specific model of beliefs that is consistent with the example in Fainmesser (2010a) is the GF model presented in the following subsection.

\subsubsection{The Global Fractions (GF) model}

For some of our results, it is useful to focus on a specific model of sellers' knowledge and beliefs with respect to the network structure, which we call the Global Fractions (GF) model. The GF model is based on the idea that the underlying process of the formation of the network has a significant random component, but that (in large networks) the fraction of sellers and buyers with a given degree is more or less constant and therefore known. ${ }^{15}$ Due to the random component, sellers have only partial information on the

\footnotetext{
${ }^{15}$ For evidence on consistency in degree distributions in large networks, see also Barabási and Albert (1999).
} 
network structure, and each seller may hold different private information about her own local area of the network.

Formally, assume that before period 1 the network $N$ is chosen u.a.r. from all of the networks with a set of sellers $S$, a set of buyers $B$, and a given degree distribution that specifies for all $d: 1$. the fraction of buyers that have degree $d$, and 2 . the fraction of sellers that have degree $d$. This underlying process of the selection of the network is common knowledge.

In addition, each seller $s$ has access to private local information including: $N_{1}(s), d_{b}$ for all $b \in N_{1}(s)$, and $R\left(b, b^{\prime}\right)$ for all $b, b^{\prime} \in N_{1}(s)$. The (Bayesian) posterior of seller $s$ is denoted by $\mathcal{D}_{G F}(s \mid N)$. We note that $\mathcal{D}_{G F}(s \mid N)$ assigns equal probability to any network satisfying 1., 2., has sets of sellers and buyers $S$ and $B$ respectively, and is consistent with the seller's private local information.

Note that specifying the aforementioned fractions is equivalent (under a simple transformation) to instead specifying the probability that the buyer (seller) in an edge $(s, b)$ selected u.a.r. from all edges of the network has degree $d$ (for all $d$ ). ${ }^{16}$ Furthermore, note that specifying $n_{b}$ along with these probabilities fully determines $n_{s}$.

Thus, for any $d_{s} \in \mathbb{Z}^{+}, \bar{b}(s) \in\left(\mathbb{Z}^{+}\right)^{d_{s}}, n_{b} \in \mathbb{Z}^{+}$and probability distributions $\mathcal{G}^{s}, \mathcal{G}^{b}$ each with bounded support on $Z^{+}$and assigning each integer a rational probability, we let $\mathcal{N}\left(d_{s}, \bar{b}(s), R, n_{b}, \mathcal{G}^{s}, \mathcal{G}^{b}\right)$ denote the set of all networks in which seller $s$ has degree $d_{s}$, the sorted vector of degrees of the buyers in $N_{1}(s)$ is equal to $\bar{b}(s)$, the reputation network $R\left(b, b^{\prime}\right)$ for all $b, b^{\prime} \in N_{1}(s)$ is given by $R$, the total number of buyers in the network is $n_{b}$, and the probability that the seller (buyer) $s^{\prime}\left(b^{\prime}\right)$ in an edge $\left(s^{\prime}, b^{\prime}\right)$ selected u.a.r. from all edges of the network has degree $d$ is equal to $\operatorname{Pr}\left(\mathcal{G}^{s}=d\right)\left(\operatorname{Pr}\left(\mathcal{G}^{b}=d\right)\right)$. Suppose further that $\operatorname{Pr}\left(\mathcal{G}^{s}=d_{s}\right)>0$, and $\operatorname{Pr}\left(\mathcal{G}^{b}=\bar{b}_{i}(s)\right)>0$ for all $i$. It is well-known that for any fixed $d_{s}, \bar{b}(s), R, \mathcal{G}^{s}, \mathcal{G}^{b}$ there exists an infinite strictly increasing sequence of integers $\left\{n_{b}\right\}$ s.t. $\mathcal{N}\left(\cdot \mid n_{b}, s\right) \neq \emptyset$. This follows from the Gale-Reyser Theorem (see e.g. Krause 1996), and (in our particular setting) Theorem 1.3 of Greenhill et al. (2006). ${ }^{17}$

\footnotetext{
${ }^{16}$ Let $P_{s}(d)$ the proportion of sellers with degree $d$, and let $\bar{d}_{s}=\sum_{d} P_{s}(d) \cdot d$ be the average sellers' degree. Then the probability that a seller $s$ in an edge that is chosen u.a.r. has degree $d$ is, $\frac{P_{s}(d) \cdot d}{\bar{d}_{s}}$.

${ }^{17}$ All statements should be read as holding only for $n_{b}$ s.t. the aforementioned set is non-empty.
} 
Consequently, if the true underlying network is $N \in \mathcal{N}\left(d_{s}, \bar{b}(s), R, n_{b}, \mathcal{G}^{s}, \mathcal{G}^{b}\right)$, then the posterior of seller $s$ is that the network is selected u.a.r. from all networks in $\mathcal{N}(\cdot)$. We denote this belief by $\mathcal{D}_{G F}(s \mid N)=\mathcal{D}_{G F}\left(d_{s}, \bar{b}(s), R, n_{b}, \mathcal{G}^{s}, \mathcal{G}^{b}\right)$.

Remark 1 The economic literature offers several models of network formation (see also Goyal 2007 and Jackson 2008 and references therein). By construction, our general model allows for any such process and captures each process via the induced distribution over the set of networks. This leaves the analysis 'formation mechanism free'. The GF model adds more structure and captures a scenario in which an individual 'has no clue' how networks are formed and therefore has a prior that is not informative about the network structure, with the exception of her knowledge of her local neighborhood, and some basic aggregate statistics. Our analysis of the GF model goes through immediately if we allow for individuals that are less informed about the network structure, and can be modified to allow for more information such as knowledge of the degrees of neighbors of neighbors and so forth. Changing the GF model beyond that will change the portion of the results that builds on the GF model.

\subsection{Cooperation networks}

We are interested in studying the interplay between the network structure $N$, sellers' knowledge and beliefs, and sellers' cooperation. In particular, we identify conditions under which the set of strategies in which all sellers always cooperate is a strict pure Bayes-Nash equilibrium of the above infinitely repeated game.

We now define what it means for a given market that is consistent with the vector $\vec{m}=\left(\pi, c, \mu, \delta,\left\{\mathcal{D}_{s}\right\}_{s \in S}\right)$ to have a totally cooperative strict Bayes-Nash equilibrium, in which case we say that $\vec{m}$ admits a cooperation network. Informally, $\vec{m}$ admits a cooperation network if for each seller $s$, given that all other sellers choose to always cooperate with all buyers connected to them, the unique best response of $s$ (under her belief distribution) is to cooperate with all buyers connected to her.

Consider a seller $s$ that has a particular belief $\mathcal{D}_{s}$ that places probability 1 on the network $N=(G, R)$, and assume that all other sellers $s^{\prime} \neq s$ always cooperate. Without loss of generality, any strategy of $s$ can be described as a mapping $f^{s}\left(t,\left\{\sigma^{\tau}\right\}_{\tau=1}^{t-1} \mid \mathcal{D}_{s}=\right.$ $N)=Q_{s}^{t}, t \geq 1$. This is true independent of whether sellers observe $\left\{\sigma^{\tau}\right\}_{t=1}^{\infty}$ or not. This follows by a simple induction since: [1] the only freedom seller $s$ has is to set her function $Q_{s}^{t}$; [2] $Q_{s}^{t}$ must be a function of the information available to seller $s$ through 
stage $t-1 ;[3]$ conditional on all other sellers $s^{\prime} \neq s$ always cooperating this information is fully captured by $\left\{Q_{s}^{\tau}\right\}_{\tau=1}^{t-1}$ and $\left\{I^{\tau}(s, b)\right\}_{b \in N_{1}(s), \tau=1 \ldots t-1} ;[4]\left\{I^{\tau}(s, b)\right\}_{b \in N_{1}(s), \tau=1 \ldots t-1}$ is deterministic given $\left\{Q_{s}^{\tau}\right\}_{\tau=1}^{t-1}$ and $\left\{\sigma^{\tau}\right\}_{\tau=1}^{t-1}$; and [5] $Q_{s}^{1}$ must be a function of $\mathcal{D}_{s}$ alone.

In fact, we can say more. Note that the periods of the repeated game are probabilistically identical until seller $s$ deviates in some interaction. Hence, there will always exist an optimal strategy in which $Q_{s}^{t}=Q_{s}^{1}$ up until the smallest $t$ such that $s$ deviates for the first time in period $t$ (which we denote by $t_{s}^{1}$ ). Similarly, denoting by $t_{s}^{k}$ the period in which seller $s$ deviates for the $k$-th time, it follows that there will always exist an optimal strategy in which $Q_{s}^{t}$ is constant for $t \in\left[1, t_{s}^{1}\right],\left(t_{s}^{1}, t_{s}^{2}\right], \ldots$.

Let $\mathcal{O}_{N}$ denote some strategy for $s$ that maximizes the expectation of her total payoff conditional on her having a particular belief $\mathcal{D}_{s}$ that places probability 1 on the network $N=(G, R)$, and assuming that all other sellers $s^{\prime} \neq s$ always cooperate. Let $\mathcal{O}_{N}(Q)$ denote the same object but with the additional restriction that $Q_{s}^{\tau}=Q$ for every $\tau \leq t_{s}^{1}$. Let $Q^{c}$ denote the strategy in which $s$ always cooperates with all buyers in all periods. Let $u_{N}(Q)$ denote the expected total payoff for $s$ due to playing strategy $Q$ conditional on her having belief $\mathcal{D}_{s}=N$, and assuming that all other sellers $s^{\prime} \neq s$ always cooperate. For ease of notation, let $\bar{u}_{N}(Q) \triangleq u_{N}\left(\mathcal{O}_{N}(Q)\right), \bar{u}_{N} \triangleq u_{N}\left(\mathcal{O}_{N}\right)$, and and $u_{N}^{c} \triangleq u_{N}\left(Q^{c}\right)$. Note that the only way in which any buyer $b$ 's trust function can be updated at the end of the first round is by setting $J_{b}^{2}(s)$ to 0 , which will happen exactly if $\sum_{b^{\prime} \in B} R\left(b, b^{\prime}\right) I^{1}\left(s, b^{\prime}\right)\left(1-Q_{s}^{1}\left(b^{\prime}\right)\right)=1$ (since $\sum_{b^{\prime} \in B} I^{1}\left(s, b^{\prime}\right) \in\{0,1\}$ ), and is equivalent to removing $(s, b)$ from the set of potential interactions. For a belief $N=(G, R)$ and a set of edges $E^{\prime}$ of $G$, let $N \backslash E^{\prime}$ denote $\left(G \backslash E^{\prime}, R\right)$. Let $\operatorname{Pr}\left(I_{N}^{t}(s, b)\right)$ denote the probability that $I^{t}(s, b)=1$ in a network $N$ conditional on $J_{b}^{t}(s)=1$ for every $(s, b) \in N$. Then by the stationarity of the game (until period $t_{s}^{1}$ ), for any belief $N$ and strategy $Q$, 


$$
\begin{aligned}
\bar{u}_{N}(Q)= & \sum_{b \in N_{1}(s): Q(b)=1} \operatorname{Pr}\left(I_{N}^{1}(s, b)\right)\left(\pi-c+\delta \cdot \bar{u}_{N}(Q)\right) \\
& +\sum_{b \in N_{1}(s): Q(b)=0} \operatorname{Pr}\left(I_{N}^{1}(s, b)\right)\left(\pi+\delta \cdot \bar{u}_{N \backslash E_{R}^{s}(b)}\right) \\
& +\left(1-\sum_{b \in N_{1}(s)} \operatorname{Pr}\left(I_{N}^{1}(s, b)\right)\right) \cdot \delta \cdot \bar{u}_{N}(Q)
\end{aligned}
$$

In particular,

$$
u_{N}^{c}=(\pi-c) \cdot \sum_{b \in N_{1}(s)} \operatorname{Pr}\left(I_{N}^{1}(s, b)\right)+\delta \cdot u_{N}^{c}
$$

It follows that,

$$
\left(\bar{u}_{N}(Q)-u_{N}^{c}\right)=\frac{\left(c+\delta \cdot\left(\bar{u}_{N \backslash E_{R}^{s}(b)}-u_{N}^{c}\right)\right) \cdot \sum_{b \in N_{1}(s): Q(b)=0} \operatorname{Pr}\left(I_{N}^{1}(s, b)\right)}{1-\delta+\delta \cdot \sum_{b \in N_{1}(s): Q(b)=0} \operatorname{Pr}\left(I_{N}^{1}(s, b)\right)}
$$

Since $\sum_{b \in N_{1}(s): Q(b)=0} \operatorname{Pr}\left(I_{N}^{1}(s, b)\right)>0$, it follows from (3) that when for every seller $s$, $\mathcal{D}_{s}$ consists of a single and fixed network $N$, the vector $\vec{m}=\left(c, \pi, \mu, \delta,\left\{\mathcal{D}_{s}\right\}_{s \in S}\right)$ admits a totally cooperative strict Bayes-Nash equilibrium if and only if for all sellers $s$ and each buyers $b \in N_{1}(s)$,

$$
c<\delta \cdot\left(u_{N}^{c}-\bar{u}_{N \backslash E_{R}^{s}(b)}(Q)\right)
$$

We now formally define a cooperation network by rephrasing these results as expectations taken over general distributions $\left\{\mathcal{D}_{s}\right\}_{s \in S}$.

\section{Definition 1 Cooperation Network:}

For a given vector $\vec{m}_{s}=\left(c, \pi, \mu, \delta, \mathcal{D}_{s}\right)$, let $I C\left(\vec{m}_{s}\right) \triangleq \min _{b \text { s.t. } \operatorname{Pr}\left(b \in N_{1}(s) \mid \mathcal{D}_{s}\right)>0} \delta\left(E_{\mathcal{D}_{s}}\left[u_{N}^{c}\right]-\right.$ $\left.E_{\mathcal{D}_{s}}\left[\bar{u}_{N \backslash E_{R}^{s}(b)}\right]\right)-c$. We refer to $I C\left(\vec{m}_{s}\right)$ as the (sufficient statistic for the) Incentives of s to Cooperate. Then,

1. We say that the vector $\vec{m}=\left(c, \pi, \mu, \delta,\left\{\mathcal{D}_{s}\right\}_{s \in S}\right)$ admits a cooperation network if and only if $\min _{s \in S} I C\left(\vec{m}_{s}\right)>0$.

2. Let $\vec{m}^{G F}(N)=\left(c, \pi, \mu, \delta,\left\{\mathcal{D}_{G F}(s \mid N)\right\}_{s \in S}\right)$. We say that a network $N$ is a $\mathrm{GF}$ cooperation network if and only if $\vec{m}^{G F}(N)$ admits a cooperation network. 


\subsection{Optimal trade networks}

We are also interested in the following design problem: for given sets of buyers and sellers, what network maximizes aggregate welfare?

Consider a market with $\bar{n}_{s}$ sellers and $\bar{n}_{b}$ buyers and parameters $c, \pi, \mu, \delta$. Let $\Delta N$ be a probability distribution over network structures $N=(G, R)$. In the unconstrained design problem, a planner chooses $\Delta N$ and compels all sellers to follow strategy $Q_{\mathcal{D}}^{c}$ (always cooperate). In the cooperation constrained design problem, the planner chooses $\Delta N$ and recommends that all sellers follow strategy $Q_{\mathcal{D}}^{c}$; sellers are then informed of $\Delta N$ and follow the planner's recommendation only if $(c, \pi, \mu, \delta, \Delta N)$ admits a cooperation network. Let $n_{b}(N)\left(n_{s}(N)\right)$ be the number of buyers (sellers) whose degree in $G$ is at least 1 . If $n_{b}(N)<\bar{n}_{b}\left(n_{s}(N)<\bar{n}_{s}\right)$ we say that $\bar{n}_{b}-n_{b}(N)$ buyers $\left(\bar{n}_{s}-n_{s}(N)\right.$ sellers $)$ are excluded from the market in $N$.

For a given network $N$, let $E[V(N)]=E\left[\sum_{s \in S} \sum_{b \in N_{1}(s)} \operatorname{Pr}\left(I_{N}^{1}(s, b)\right)\right]$ denote the expected volume of trade (number of transactions) in good $H$ that is achieved in a given period if all sellers follow strategy $Q_{\mathcal{D}}^{c}$. Denote by $E[V(\Delta N)]$ the corresponding value given a probability distribution $\Delta N$ over networks. Let $N \in \mathcal{N}\left(\bar{n}_{b}, \bar{n}_{s}\right)$ if $n_{b}(N) \leq \bar{n}_{b}$ and $n_{s}(N) \leq \bar{n}_{s}$, and let $N^{u c}\left(\bar{n}_{b}, \bar{n}_{s}, c, \pi, \mu, \delta\right)\left(N^{c}\left(\bar{n}_{b}, \bar{n}_{s}, c, \pi, \mu, \delta\right)\right)$ be the solution to the unconstrained (constrained) design problem. Then,

$$
N^{u c}\left(\bar{n}_{b}, \bar{n}_{s}, c, \pi, \mu, \delta\right)=\underset{\Delta N \mid \operatorname{supp}(\Delta N) \subseteq \mathcal{N}\left(\bar{n}_{b}, \bar{n}_{s}\right)}{\operatorname{argmax}} E[V(\Delta N)]
$$

and

$$
N^{c}\left(\bar{n}_{b}, \bar{n}_{s}, c, \pi, \mu, \delta\right)=\underset{\Delta N \mid \operatorname{supp}(\Delta N) \subseteq \mathcal{N}\left(\bar{n}_{b}, \bar{n}_{s}\right)}{\operatorname{argmax}} \quad \underset{, \min _{s \in S} I C(c, \pi, \mu, \delta, \Delta N)>0}{E[V(\Delta N)]},
$$

where $\min _{s \in S} I C(c, \pi, \mu, \delta, \Delta N)>0$ guarantees that $(c, \pi, \mu, \delta, \Delta N)$ admits a cooperation network.

Thus, the proportion of welfare loss due to the constraints on the structure of cooperation networks is

$$
W L\left(\bar{n}_{b}, \bar{n}_{s}, c, \pi, \mu, \delta\right)=1-\frac{E\left[V\left(N^{c}\left(\bar{n}_{b}, \bar{n}_{s}, c, \pi, \mu, \delta\right)\right)\right]}{E\left[V\left(N^{u c}\left(\bar{n}_{b}, \bar{n}_{s}, c, \pi, \mu, \delta\right)\right)\right]}
$$

If $W L\left(\bar{n}_{b}, \bar{n}_{s}, c, \pi, \mu, \delta\right)=0$, then cooperation networks achieve the first best in a market with $\left(\bar{n}_{b}, \bar{n}_{s}, c, \pi, \mu, \delta\right)$. 


\section{Main results}

\subsection{Cooperation based on local beliefs}

Our first result, which provides the foundation for later results, shows that whether or not a given vector $\vec{m}$ admits a cooperation network is asymptotically independent of the sellers' global beliefs, and depends only on their local beliefs. We do that by showing that a seller can determine whether or not to cooperate given only local information about the network, making our model computationally (and analytically) attractive. This is quite surprising, since the fact that we focus on networks with bounded degree implies that the overwhelming majority of information about other buyers and sellers is not included in a seller's local beliefs.

Theorem 1 Local Beliefs Theorem:

For any fixed $c, \pi, \mu, \delta, \Delta \in \mathbb{Z}^{+}$, and $\epsilon>0$, there exists a finite constant $d=d(c, \pi, \mu, \delta, \Delta, \epsilon)$ independent of the size of the entire network s.t. for any belief $\mathcal{D}_{s}$ satisfying $\operatorname{deg}\left(\mathcal{D}_{s}\right) \leq$ $\Delta$,

$$
\left|I C\left(c, \pi, \mu, \delta, \mathcal{D}_{s}\right)-I C\left(c, \pi, \mu, \delta, \mathcal{D}_{s}^{d}\right)\right|<\epsilon .
$$

Theorem 1 implies that whenever we can make comparative statements about cooperation under beliefs $\left\{\mathcal{D}_{s}^{d}\right\}_{s \in S}$, we can also make (asymptotic) comparative statements about cooperation under beliefs $\left\{\mathcal{D}_{s}\right\}_{s \in S}$.

A special case of Theorem 1 is when sellers have complete knowledge of the true underlying network. Formally, consider a true network $N$, and for every $s \in S$, let $\mathcal{D}_{s}$ place probability 1 on $N$. In this case, an implication of Theorem 1 is that whenever we can make comparative statements about cooperation given the local neighborhoods of all sellers (i.e. $\left\{N_{d}(s)\right\}_{s \in S}$ ), we can also make (asymptotic) comparative statements for the entire network $N$. This later interpretation of Theorem 1 suggests that when a seller $s$ determines whether or not to cooperate she "discounts" links that are at a large distance from her and can asymptotically do as good by considering only her local neighborhood. 
The proof of Theorem 1 builds on recent developments in graph theory, and in particular on GG who study randomized greedy algorithms for matchings in a graph, and the relationship between the local and global properties of the set of matchings of a graph.

Proof. We prove Theorem 1 for the following restricted domain of seller's beliefs: for any seller $s$ the belief $\mathcal{D}_{s}$ is such that $\operatorname{Pr}\left(b \in N_{1}(s) \mid \mathcal{D}_{s}\right) \in\{0,1\}$. Namely, for any $b \in B$, either seller $s$ believes that she is connected to $b$ with probability 1 , or she believes (with probability 1 ) that she is not connected to $b$. The proof of the case that $\operatorname{Pr}\left(b \in N_{1}(s) \mid \mathcal{D}_{s}\right) \in[0,1]$ follows the same argument but requires additional notation and is omitted.

Now, let us fix any network $N$, and let $N(s, d)$ denote the depth-d neighborhood of seller $s$ in $N$. Note that one may interpret the quantity $\operatorname{Pr}\left(I_{N}^{1}(s, b)\right)\left(\operatorname{Pr}\left(I_{N(s, d)}^{1}(s, b)\right)\right)$ as the probability that edge $(s, b)$ is chosen to belong to the random graph matching constructed by examining the edges of $N(N(s, d))$ (with each seller deleted w.p. $\mu$ ) in a random order (selected u.a.r.) and including an edge if no incident edges have already been examined. Noting that this randomized greedy matching construction is identical to the matching algorithm GREEDY studied in GG, it follows from Lemma 6 of GG and a simple conditioning argument (to account for $\mu$ ) that

\section{Lemma 1 Locality Lemma:}

For any fixed $c, \pi, \mu, \delta, \Delta \in \mathbb{Z}^{+}$, and $\epsilon>0$, there exists a finite constant $d=d(c, \pi, \mu, \delta, \Delta, \epsilon)$ independent of the size of the entire buyer-seller network s.t. for any network $N$ satisfying $\operatorname{deg}(N) \leq \Delta$,

$$
\left|\operatorname{Pr}\left(I_{N}^{1}(s, b)\right)-\operatorname{Pr}\left(I_{N(s, d)}^{1}(s, b)\right)\right|<\epsilon .
$$

Theorem 1 - proof (cont.): Second, Equations (1) and (2) imply that

$$
u_{N}^{c}=(\pi-c) \cdot \frac{\sum_{b \in N_{1}(s)} \operatorname{Pr}\left(I_{N}^{1}(s, b)\right)}{1-\delta}
$$


and

$$
\begin{aligned}
\bar{u}_{N}= & \max _{Q}\left[\bar{u}_{N}(Q)\right]= \\
= & \max _{Q}\left(\frac{\pi \sum_{b \in N_{1}(s)} \operatorname{Pr}\left(I_{N}^{1}(s, b)\right)-c \sum_{b \in N_{1}(s): Q(b)=1} \operatorname{Pr}\left(I_{N}^{1}(s, b)\right)}{1-\delta+\delta \sum_{b \in N_{1}(s): Q(b)=0} \operatorname{Pr}\left(I_{N}^{1}(s, b)\right)}\right. \\
& \left.\quad+\frac{\delta \sum_{b \in N_{1}(s): Q(b)=0} \operatorname{Pr}\left(I_{N}^{1}(s, b)\right)}{1-\delta+\delta \sum_{b \in N_{1}(s): Q(b)=0} \operatorname{Pr}\left(I_{N}^{1}(s, b)\right)} \cdot \bar{u}_{N \backslash E_{R}^{s}(b)}\right)
\end{aligned}
$$

Finally, noting that in a network of maximum degree $\Delta$, one has $\operatorname{Pr}\left(I_{N}^{1}(s, b)\right) \in$ $\left[\frac{1}{2 \Delta-1}, 1\right]$ for all edges $(s, b)$, Theorem 1 follows by interpreting Equation (5) as a dynamic program (for computing $\bar{u}_{N}$ ), combined with Lemma 1 and a simple induction, and applying the same logic to (4).

\subsection{Cooperation and network structure in the GF model}

For given $\pi, c, \delta$, and $\mu$, a key question is: what network structures have a totally cooperative strict Bayes-Nash equilibrium? In this section, we focus on sellers whose knowledge and beliefs are consistent with the GF model and derive conditions on a network $N$ such that $N$ is a GF cooperation network.

We first show that for all finite support distributions $\mathcal{G}^{s}, \mathcal{G}^{b}$ and for every seller $s$ in any asymptotically large network $N$ with degree distributions $\mathcal{G}^{s}, \mathcal{G}^{b}$, the belief of seller $s\left(\mathcal{D}_{G F}(s \mid N)\right)$ is asymptotically identical to the belief that the network looks locally like a corresponding simple random tree.

For given $d_{0} \geq 0, \bar{b} \in\left(Z^{+}\right)^{d_{0}}$, distributions $\mathcal{G}^{s}, \mathcal{G}^{b}$ with finite support on $\mathbb{Z}^{+}$, and $d \geq 1$, let $T\left(d_{0}, \bar{b}, R, \mathcal{G}^{s}, \mathcal{G}^{b}, d\right)$ denote the random depth-d tree such that the root $r$ has degree $d_{0}$, the sorted vector of degrees of the children of $r$ is $\bar{b}$, all subsequent nonleaf nodes at an even depth have a number of children drawn i.i.d. from $\mathcal{G}^{s}-1$, all subsequent non-leaf nodes at an odd depth have a number of children drawn i.i.d. from $\mathcal{G}^{b}-1$, the underlying reputation network for $N_{1}(s)$ is $R$, and for all other buyers pairs $\left(b, b^{\prime}\right), R\left(b, b^{\prime}\right)$ is 1 with probability (w.p.) 0.5 , and 0 w.p. 0.5 . Then we have

Lemma 2 Locally Tree-Like Lemma: 
For all $d_{0}, \bar{b}, R$, and finite support $\mathcal{G}^{s}, \mathcal{G}^{b}$, and for all $d>0$,

$$
\lim _{n_{b} \rightarrow \infty} T V D\left(\mathcal{D}_{G F}^{d}\left(d_{0}, \bar{b}, R, n_{b}, \mathcal{G}^{s}, \mathcal{G}^{b}\right), T\left(d_{0}, \bar{b}, R, \mathcal{G}^{s}, \mathcal{G}^{b}, d\right)\right)=0
$$

The proof of Lemma 2 is presented in Appendix A. In particular, the key to proving Lemma 2 is the so-called configuration method (see Wormald 1999). Using this technique, a random graph is related to a different random object - the configuration model. In the configuration model, each buyer is viewed as a bucket, and each seller is viewed as a bucket. Each bucket is endowed with a number of points equal to the desired degree of the corresponding buyer (seller). The points in the buckets are then matched randomly, and a seller and buyer are connected if a point from that seller bucket is matched to a point of the corresponding buyer bucket. There is a vast literature that both analyzes the configuration model, and relates it back to many random-graph models of interest (see e.g. Greenhill et al. 2006, Wormald 1999). Such relations often involve subtle counting and conditioning arguments - for more details the reader is referred to the proof in Appendix A. We note that results of this nature are often quite useful, since it may be easier to develop intuition about a network which looks locally tree-like.

Lemma 2 implies that in a large network $N$, the sequence of beliefs $\mathcal{D}_{G F}^{d}\left(d_{0}, \bar{b}, R, n_{b}, \mathcal{G}^{s}, \mathcal{G}^{b}\right)$, $d=1,2, \ldots$, converges in a sense to a belief on an 'infinite tree'. As a result, in a large network $N$, and as long as sellers' beliefs are consistent with the GF model, many relevant quantities can be described in terms of the associated limits. Most relevant for the characterization of large cooperation networks, Theorem 2 establishes the connection between the incentives of seller $s$ to cooperate with buyer $b$ in a network $N$ and the corresponding incentives in the corresponding 'infinite random tree'.

Theorem 2 Asymptotic Characterization of Cooperation Networks I:

For all $c, \pi, \mu, \delta, d_{0}, \bar{b}$, and finite support $\mathcal{G}^{s}, \mathcal{G}^{b}, \lim _{d \rightarrow \infty} I C\left(c, \pi, \mu, \delta, T\left(d_{0}, \bar{b}, R, \mathcal{G}^{s}, \mathcal{G}^{b}, d\right)\right)$ and $\lim _{n_{b} \rightarrow \infty} I C\left(c, \pi, \mu, \delta, \mathcal{D}_{G F}\left(d_{0}, \bar{b}, R, n_{b}, \mathcal{G}^{s}, \mathcal{G}^{b}\right)\right)$ both exist, and equal one-another.

Proof. The theorem follows from Theorem 1 and Lemma 2.

Theorem 2 implies an explicit asymptotic characterizations of cooperation networks 
in terms of a dynamic program that is based on the belief that the network is a random tree. Moreover, in a particularly interesting limit case we can give an especially simple characterization.

Let $R^{1}$ be a reputation network such that for all $b^{\prime}$ and $b, R^{1}\left(b^{\prime}, b\right)=1$. We say that $R^{1}$ is the complete reputation network which captures a situation in which each buyer shares information with all other buyers.

Corollary 1 Asymptotic Characterization of Cooperation Networks II:

Let $c, \pi, \mu, \delta, d_{0}, \bar{b}, \mathcal{G}^{s}, \mathcal{G}^{b}$ be fixed and $\mathcal{G}^{s}, \mathcal{G}^{b}$ have finite support. For a seller $s$, let $N_{d, s}$ denote the belief $T\left(d_{0}, \bar{b}, R, \mathcal{G}^{s}, \mathcal{G}^{b}, d\right)$. Then $\lim _{d \rightarrow \infty} \sum_{b \in N_{1}(s)} \operatorname{Pr}\left(I_{N_{d, s}}^{1}(s, b)\right)$ exists, and $\operatorname{sign}\left(\lim _{n_{b} \rightarrow \infty} I C\left(c, \pi, \mu, \delta, \mathcal{D}_{G F}\left(d_{0}, \bar{b}, R^{1}, n_{b}, \mathcal{G}^{s}, \mathcal{G}^{b}\right)\right)\right)=\operatorname{sign}\left(\frac{\delta(\pi-c)}{1-\delta} \lim _{d \rightarrow \infty} \sum_{b \in N_{1}(s)} \operatorname{Pr}\left(I_{N_{d, s}}^{1}(s, b)\right)-c\right)$.

Proof. That the necessary limit exists follows from Lemma 1. To derive (6) we first describe how Equation (3) simplifies in the special case where the reputation network is captured by $R^{1}$. Since in this case $\bar{u}_{N \backslash E_{R}^{s}(b)}=0$ for all $b$, it follows from (3) that for any fixed belief $N$ and strategy $Q$,

$$
\left(\bar{u}_{N}(Q)-u_{N}^{c}\right)=\frac{\left(c-\delta \cdot u_{N}^{c}\right) \cdot \sum_{b \in N_{1}(s): Q(b)=0} \operatorname{Pr}\left(I_{N}^{1}(s, b)\right)}{1-\delta+\delta \cdot \sum_{b \in N_{1}(s): Q(b)=0} \operatorname{Pr}\left(I_{N}^{1}(s, b)\right)}
$$

The Corollary then follows from (7), (4), and Theorem 2.

We now use Corollary 1 and the corresponding tree structure described in Lemma 2 and Theorem 2 to make comparative statements about the structure of cooperation network.

\subsection{Third-party observability, network structure, and cooperation}

In this section, we focus on the following question: how do the patterns of third party observability, as captured by the reputation component of the network $(R)$, affect the ability of different patterns of repeated interactions, as captured by the buyer-seller component of the network $(G)$, to facilitate cooperation? We first establish that third-party observability helps cooperation and allows a bigger set of buyer-seller networks to sustain cooperation. The intuition is consistent with much of the literature on community 
enforcement; when more buyers are aware of a seller's deviation, the seller faces a larger punishment for deviating.

Proposition 1 Weak Monotonicity of Cooperation in Third-Party Observability:

Let $c, \pi, \mu, \delta, d_{0}, \bar{b}, \mathcal{G}^{s}, \mathcal{G}^{b}$ be fixed and $\mathcal{G}^{s}, \mathcal{G}^{b}$ have finite support, and let $I C_{\mathcal{D}_{G F}}\left(R, n_{b}\right)=I C\left(c, \pi, \mu, \delta, \mathcal{D}_{G F}\left(d_{0}, \bar{b}, R, n_{b}, \mathcal{G}^{s}, \mathcal{G}^{b}\right)\right)$. Then, for any reputation network $R, I C_{\mathcal{D}_{G F}}\left(R, n_{b}\right) \leq I C_{\mathcal{D}_{G F}}\left(R^{1}, n_{b}\right) .{ }^{18}$

Proof. By definition, $I C_{\mathcal{D}_{G F}}\left(R, n_{b}\right)=\min _{b \in N_{1}(s)} \delta\left(E_{\mathcal{D}_{G F}\left(d_{0}, \bar{b}, R, n_{b}, \mathcal{G}^{s}, \mathcal{G}^{b}\right)}\left[u_{N}^{c}\right]-E_{\mathcal{D}_{G F}\left(d_{0}, \bar{b}, R, n_{b}, \mathcal{G}^{s}, \mathcal{G}^{b}\right)}\left[\bar{u}_{N \backslash E_{R}^{s}(b)}\right]\right)-c$.

The lemma then follows from the fact that: [1] For all $R, E_{\mathcal{D}_{G F}\left(d_{0}, \bar{b}, R, n_{b}, \mathcal{G}^{s}, \mathcal{G}^{b}\right)}\left[\bar{u}_{N \backslash E_{R}^{s}(b)}\right] \geq$ $0 ;[2] E_{\mathcal{D}_{G F}\left(d_{0}, \bar{b}, R^{1}, n_{b}, \mathcal{G}^{s}, \mathcal{G}^{b}\right)}\left[\bar{u}_{N \backslash E_{R^{1}}^{s}(b)}\right]=0$; and $[3] E_{\mathcal{D}_{G F}\left(d_{0}, \bar{b}, R, n_{b}, \mathcal{G}^{s}, \mathcal{G}^{b}\right)}\left[u_{N}^{c}\right]=$ $E_{\mathcal{D}_{G F}\left(d_{0}, \bar{b}, R^{1}, n_{b}, \mathcal{G}^{s}, \mathcal{G}^{b}\right)}\left[u_{N}^{c}\right]$.

An implication of Proposition 1 is that the complete reputation network $R^{1}$ allows for the largest set (in the sense of set inclusion) of buyer-seller cooperation networks $(G)$ for any $c, \pi, \mu, \delta$.

Note that ideally one would like to prove a stronger form of monotonicity of cooperation in third-party observability. Namely, that adding links to a reputation networks always weakly improves cooperation. We conjecture that this claim is also true, but cannot provide a proof at this stage.

We now focus on networks that take the form $N=\left(G, R^{1}\right)$ and make comparative statements with respect to the incentives of sellers to cooperate as a function of $G$. Given Proposition 1, this exercise provides insights on the limits of cooperation and on the types of inefficiencies that can (and those that cannot) be circumvented by improving observability in a market.

A fundamental observation that is captured by Corollary 1 is that when $R=R^{1}$, sellers who expect to sell with higher overall probability (larger $\sum_{b \in N_{1}(s)} \operatorname{Pr}\left(I_{N}^{1}(s, b)\right)$ ) have stronger incentives to cooperate. As a result, cooperation is better sustained when sellers' (buyers') degrees are large (small). To see why, note that [1] the probability that a seller $s$ sells in a given period is an increasing function of her degree; and [2] the

\footnotetext{
${ }^{18}$ Proposition 1 extends immediately to the general belief model $\left(\mathcal{D}_{s}\right)$.
} 
probability that $s$ sells in a given period is an increasing function of the degree of any seller that is connected to a buyer that $s$ is connected to. The latter is true because sellers with high degrees are less likely to sell to each one of the buyers to whom they are connected. More generally, when sellers' degrees are large and buyers' degrees are small, there are more buyers and less sellers that can be involved in trade. Consequently, sellers sell with high probability and expect large payoffs. Figure 1 provides a simple deterministic example of the general rule that is captured by Proposition 2.

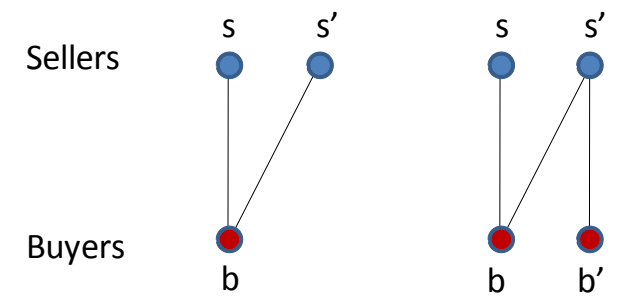

Figure 1: Consider the two networks above and assume that $\mu=1$ and that in the rightmost network buyer $b$ and buyer $b^{\prime}$ are connected and share information on their past transactions with seller $s^{\prime}$. In the leftmost network, seller $s$ expects to sell a good with probability $\frac{1}{2}$ in any given period. As a result, conditional on cooperation between $s^{\prime}$ and $b$, seller $s$ cooperates with $b$ if and only if $\frac{\delta}{1-\delta}(\pi-c) \cdot \frac{1}{2}>c$. In the rightmost network, seller $s^{\prime}$ has an additional link. Clearly, this increases the probability that seller $s^{\prime}$ sells in a given period. However, this also increases the probability that seller $s$ sells in a given period from $\frac{1}{2}$ to $\frac{2}{3}$. As a result, conditional on cooperation between $s^{\prime}$ and $b$, as well as between $s^{\prime}$ and $b^{\prime}$, seller $s$ cooperates with $b$ if and only if $\frac{\delta}{1-\delta}(\pi-c) \cdot \frac{2}{3}>c$.

\section{Proposition 2 Monotonicity of Cooperation in Degree:}

Let $c, \pi, \mu, \delta$ be fixed. Suppose that for all $d \geq 1$, the random tree $T^{2}=$

$T\left(d_{0}^{2}, \bar{b}^{2}, R^{1}, \mathcal{G}^{2^{s}}, \mathcal{G}^{2^{b}}, d\right)$ can be constructed (on the same probability space) from the random tree $T^{1}=T\left(d_{0}^{1}, \bar{b}^{1}, R^{1}, \mathcal{G}^{1^{s}}, \mathcal{G}^{1^{b}}, d\right)$ by performing only the two operations: [1] appending (as children) subtrees to seller nodes in an arbitrary way, and [2] removing (as children) subtrees from buyer nodes in an arbitrary way. Then,

$\lim _{n_{b} \rightarrow \infty} I C\left(c, \pi, \mu, \delta, \mathcal{D}_{G F}\left(d_{0}^{1}, \bar{b}^{1}, R^{1}, n_{b}, \mathcal{G}^{1^{s}}, \mathcal{G}^{1^{b}}\right)\right)>0$ implies

$\lim _{n_{b} \rightarrow \infty} I C\left(c, \pi, \mu, \delta, \mathcal{D}_{G F}\left(d_{0}^{2}, \bar{b}^{2}, R^{1}, n_{b}, \mathcal{G}^{2^{s}}, \mathcal{G}^{2^{b}}\right)\right)>0$.

Proof. Consider a randomized matching algorithm that progresses by examining the edges of a network in a random order (selected u.a.r.) and including an edge if no incident edges have already been examined. GG study the properties of this algorithm 
which they name GREEDY. It will be useful first state an important monotonicity property of GREEDY, which follows from Proposition 1 of GG and a straightforward induction/coupling argument.

Lemma 3 Suppose that $\widehat{G}, G$ are (rooted) tree networks, and $\widehat{G}$ can be constructed from $G$ by performing only the two operations: [1] appending (as children) subtrees to nodes at even depth in $G$ in an arbitrary way, and [2] removing (as children) subtrees from nodes at odd depth in $G$ in an arbitrary way (where the depth of the root is 0 by default). Then the probability that GREEDY matches the root of $\widehat{G}$ when run on $\widehat{G}$ is at least the probability that GREEDY matches the root of $G$ when run on $G$.

The proof of Proposition 2 then follows from Lemma 3 and interpreting $\operatorname{Pr}\left(I_{N}^{1}(s, b)\right)$ as the probability that edge $(s, b)$ is selected by GREEDY.

We note that to some extent the opposite occurs in networks with sparse reputation networks, but we do not formalize that here. ${ }^{19}$. The perfect alignment between a seller's probability of selling and her incentives to cooperate have an additional, more powerful implication: consider two networks $N^{\prime}=\left(G^{\prime}, R^{1}\right)$ and $N^{\prime \prime}=\left(G^{\prime \prime}, R^{1}\right)$, and assume that conditional on full cooperation, the minimal probability of selling of any seller is weakly higher in $N^{\prime \prime}$ than in $N^{\prime}\left(\min _{s} \sum_{b \in N_{1}^{\prime \prime}(s)} \operatorname{Pr}\left(I_{N^{\prime \prime}}^{1}(s, b)\right) \geq \min _{s} \sum_{b \in N_{1}^{\prime}(s)} \operatorname{Pr}\left(I_{N^{\prime}}^{1}(s, b)\right)\right)$. Then $N^{\prime}$ being a cooperation network implies that $N^{\prime \prime}$ is a cooperation network.

In networks in which all sellers are symmetric, the perfect alignment between the probability of selling and the incentives to cooperate implies that the expected aggregate volume of trade and the incentives of a seller to cooperate are aligned. We now demonstrate this insight using a special family of buyer-seller networks which we call semi-regular networks. In a semi-regular network all sellers have the same degree $d_{S}$ and all buyers have the same degree $d_{B}$. We also show that adding a sufficiently large number of links to a buyer-seller network $(G)$ guarantees that: [1] if $R=R^{1}$, sellers have (asymptotically) the maximal incentives to cooperate possible given $n_{s}, n_{b}$; and [2] conditional on $N$ being a cooperation network, the expected number of transactions

\footnotetext{
${ }^{19}$ Fainmesser (2010a) characterizes the structure of cooperation networks when the reputation network is given by $R^{0}$ - a reputation network such that for all $b^{\prime} \neq b, R^{0}\left(b^{\prime}, b\right)=0$.
} 
in every period is (asymptotically) maximal given $n_{s}, n_{b}$. The proof of Theorem 3 is presented in Appendix B.

Theorem 3 Dense Networks Maximize Welfare and Incentives to Cooperate:

Suppose there exist positive integers $d_{S}, d_{B}$ s.t. $\operatorname{Pr}\left(d_{s}=d_{S} \mid \mathcal{D}_{G F}\right)=1, \operatorname{Pr}\left(d_{b}=d_{B} \mid \mathcal{D}_{G F}\right)=$ 1. Then,

$$
\operatorname{sign}\left(I C\left(c, \pi, \mu, \delta, \mathcal{D}_{G F}\left(R^{1}, n_{b}, d_{S}, d_{B}\right)\right)=\operatorname{sign}\left(\frac{\delta(\pi-c)}{1-\delta} \cdot \frac{1}{n_{s}} \cdot E\left[V\left(\mathcal{D}_{G F}\left(R^{1}, n_{b}, d_{S}, d_{B}\right)\right)\right]-c\right)\right.
$$

and

$$
\lim _{n_{b} \rightarrow \infty} \frac{E\left[V\left(\mathcal{D}_{G F}\left(R^{1}, n_{b}, d_{S}, d_{B}\right)\right]\right.}{\min \left(\mu \cdot n_{b} \cdot \frac{d_{B}}{d_{S}}, n_{b}\right)} \geq 1-\left(\max \left(d_{S}, d_{B}\right)-1\right)^{-1} .
$$

Moreover, $\frac{n_{b}}{n_{s}}=\frac{d_{S}}{d_{B}}$. Thus, $\min \left(\mu \cdot n_{b} \cdot \frac{d_{B}}{d_{S}}, n_{b}\right)=\min \left(\mu \cdot n_{s}, n_{b}\right)$, which equals the maximal volume of trade possible conditional on $n_{b}$ and $n_{s} \cdot{ }^{20}$

Theorem 3 implies an asymptotic lower bound on the incentives to cooperate.

$$
\lim _{n_{b} \rightarrow \infty} I C\left(c, \pi, \mu, \delta, \mathcal{D}_{G F}\left(R^{1}, n_{b}, d_{S}, d_{B}\right)\right) \geq \frac{\delta(\pi-c)}{1-\delta} \cdot \min \left(\mu, \frac{d_{S}}{d_{B}}\right) \cdot\left(1-\left(\max \left(d_{S}, d_{B}\right)-1\right)^{-1}\right)-c .
$$

Expression (8) highlights the importance of the ratio $\frac{d_{S}}{d_{B}}$ (or $\frac{n_{b}}{n_{s}}$ ) in determining whether repeated interactions can sustain cooperation in a market that gives positive probability for any of $n_{s}$ sellers to sell and any of $n_{b}$ buyers to buy. If the ratio $\frac{n_{b}}{n_{s}}$ is large enough, cooperation can asymptotically be sustained and maximal volume of trade facilitated even with limited (yet large) degrees. Corollary 2 shows that Theorem 3 has implications to welfare that go beyond the semi-regular setup.

Recall that $W L\left(\bar{n}_{b}, \bar{n}_{s}, c, \pi, \mu, \delta\right)$ is defined in section 2.4 as the proportion of welfare loss due to the constraints on the structure of cooperation networks. Recall further that if $\frac{\delta(\pi-c)}{1-\delta} \cdot \mu-c<0$ then no network (apart from the empty network) is a cooperation network. This is true because even a seller who is guaranteed to sell whenever she produces, and expects to lose her entire future payoff if she sells good $L$, will still

\footnotetext{
${ }^{20}$ From an algorithmic perspective it is interesting to note that in addition to being easy to implement and leading to a tractable analysis, the simple matching mechanism governing our buyer-seller market is also asymptotically welfare maximizing w.r.t. the volume of trade when all sellers cooperate, and all sellers (buyers) have the same (large) degree $d_{S}\left(d_{B}\right)$. This is surprising, since the mechanism is quite simplistic, and corresponds better to a random decentralized market than to known algorithms for constructing optimal matchings.
} 
deviate. Consequently, $\frac{\delta(\pi-c)}{1-\delta} \cdot \mu-c<0$ implies that $W L\left(n_{b}, n_{s}, c, \pi, \mu, \delta\right)>0$ for any $n_{b}, n_{s}$. Corollary 2 covers the more interesting case where $\frac{\delta(\pi-c)}{1-\delta} \cdot \mu-c>0$. The proof is deferred to Appendix B.

\section{Corollary 2 Barrier to Entry and Asymptotic Efficiency:}

Consider a market with $\bar{n}_{b}$ buyers and $\gamma \bar{n}_{b}$ sellers, and fixed $c, \pi, \delta$ s.t. $\delta \in[0,1)$. Let $\frac{\delta(\pi-c)}{1-\delta}-c>0$. Then,

1. For any $\gamma, W L\left(\bar{n}_{b}, \gamma \bar{n}_{b}, c, \pi, \mu=1, \delta\right)=0$.

2. Let $\mu<1$. There exists $\bar{\gamma}(\mu)$ such that $W L\left(\bar{n}_{b}, \gamma \bar{n}_{b}, c, \pi, \mu, \delta\right)>0$ for any $\gamma>\bar{\gamma}$.

3. For any $\mu \in\left[c \cdot \frac{1-\delta}{\delta(\pi-c)}, 1\right]$ and $\gamma \in \mathbb{Q}^{+}$

$$
\lim _{\bar{n}_{b} \rightarrow \infty} W L\left(\bar{n}_{b}, \gamma \bar{n}_{b}, c, \pi, \mu, \delta\right)=0
$$

Part 1 of Corollary 2 asserts that when there is no stochastic element in the production technology $(\mu=1)$, the incentive constraints do not restrict welfare. In particular, when $\mu=1$, a network that consists of pairs of buyers and sellers and some excluded buyers or sellers (but not both) provides the maximal volume of trade as well as the maximal incentives to cooperate. On the other hand, part 2 of the Corollary addresses the case of stochastic production technology. If $\mu<1$ the maximal volume of trade cannot be achieved if any seller is excluded from the market. At the same time, if there are many more sellers than buyers, all cooperation networks exclude some sellers from the market. This leads to a welfare loss. Figure 2 provides an example.

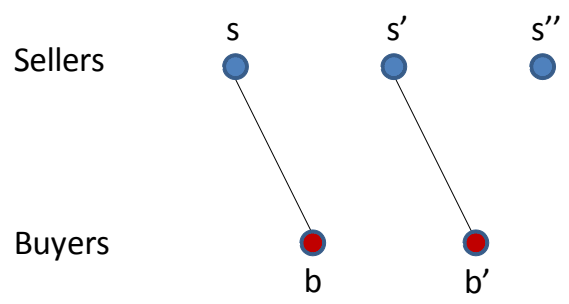

Figure 2: Assume that $\mu=1$. In the above network, seller $s$ cooperates with buyer $b$ (and seller $s^{\prime}$ cooperates with buyer $b^{\prime}$ ), if and only if $\frac{\delta}{1-\delta}(\pi-c)>c$. Moreover, conditional on cooperation between every buyer and seller that are connected, two goods will be sold in every period. This is the maximal number of goods that can be sold in one period in a network with three sellers and two buyers. Now assume that $\mu<1$. There exists positive probability that in a given period only sellers $s$ and $s^{\prime \prime}$ are active. Thus, any network in 
which seller $s^{\prime \prime}$ is not connected to any buyer limits the number of trades to less than two even though two sellers are active.

Part 3 of Corollary 2 is encouraging; in large markets (asymptotic) efficiency is restored. Theorem 3 provides the necessary intuition: let $d_{S}=\frac{d_{B}}{\gamma^{\prime}}$ and fix $\gamma^{\prime}$, then as long as $1 \geq \gamma^{\prime} \cdot \mu$,

$$
\lim _{n_{b} \rightarrow \infty} \lim _{d_{B} \rightarrow \infty} I C\left(\mathcal{D}_{G F}\left(R^{1}, n_{b}, d_{S}, d_{B}\right)\right)=\frac{\delta(\pi-c)}{1-\delta} \cdot \mu-c
$$

and as long as $1 \leq \gamma^{\prime} \cdot \mu$,

$$
\lim _{n_{b} \rightarrow \infty} \lim _{d_{B} \rightarrow \infty} \frac{E\left[V\left(\mathcal{D}_{G F}\left(R^{1}, n_{b}, d_{S}, d_{B}\right)\right)\right]}{n_{b}}=1
$$

Going back to part 3 of Corollary 2, no matter how large is $\frac{\bar{n}_{s}}{\bar{n}_{b}}$, a planner can choose $\Delta N$ in the following way: [1] set $R=R^{1}$; and [2] pick large positive integers $d_{S}, d_{B}$ s.t. $\frac{d_{S}}{d_{B}}=\mu$ and choose $G$ u.a.r. from the set of buyer-seller networks s.t. $\operatorname{Pr}\left(d_{b}=d_{B}\right)=1$, $\operatorname{Pr}\left(d_{s}=d_{S}\right)=\frac{\bar{n}_{b}}{\bar{n}_{s}} \cdot \frac{1}{\mu}$, and $\operatorname{Pr}\left(d_{s}=0\right)=1-\frac{\bar{n}_{b}}{\bar{n}_{s}} \cdot \frac{1}{\mu}$. Then, the planner achieves (asymptotically in $d_{S}, d_{B}$ ) both high incentives to cooperate and maximal volume of trade. This is interesting as the planner does not need to create a complete network. In fact, $d_{B}\left(d_{S}\right)$ does not need to be in the order of $n_{s}\left(n_{b}\right)$ and can be much smaller. The implications of our results in the context of barriers to entry and efficiency are discussed further in section 4.3 .

\section{Discussion}

In this section, we first highlight the implications of our methodological contribution to social networks analysis in economics, sociology, and psychology. Then, we provide an interpretation of our characterization of cooperation networks in a more traditional context of market structure and discuss the implications for barriers to entry and efficiency.

\subsection{The (un)importance of global beliefs}

The recent literature on static network games suggests that when players have incomplete knowledge of the networks structure, the analysis of the induced (Bayesian) game is much simpler than the corresponding analysis when players know the entire network 
structure. Galeotti et al. (2010) summarize that "the key insight is that when players have limited information about the network they are unable to condition their behavior on its fine details and this leads to a significant simplification". However, this does not mean that global knowledge of the network is not important. In fact, Galeotti et al. provide several examples in which changing the information structure changes the set of equilibria significantly.

Kets (2010) shows that when a game is local and players have a common prior, there are weak conditions under which small changes to the priors do not change the equilibrium payoffs. While it is not straightforward to define small changes in priors over the network, Kets defines small changes for the family of static network games studied by Galeotti et al.

Theorem 1 provides a first stab at evaluating the importance of different changes to players' priors in the context of repeated games in networks. In our model, for the family of totally cooperative equilibria, any change to a belief of a seller that keeps the seller's belief over her local neighborhood intact is a small change in the sense that it does not affect the seller's best response correspondence. In that sense, global beliefs are strategically unimportant.

The result that global beliefs are strategically unimportant is also true for many static network games. However, the problem studied here is more complex. Due to the dynamic nature of the interactions and the limited supply and demand, a seller's incentives to cooperate generally depend on the entire network structure even if she conditions on the actions taken by all of the buyers and sellers in the network. This makes the result even more surprising and suggests that similar results may be derived even when the patterns of interaction are complex.

Moreover, the methodology used in this paper can be applied to other setups. For example, Theorem 1 can be easily modified to include networks that are not bipartite and allow for a model in which agents can be buyers in some periods and sellers in others. 


\subsection{Random network formation and random trees}

The following three ideas raise separate interest in economics, sociology, and psychology: [1] the formation process of many social networks has a strong stochastic component; [2] individuals do not know the exact structure of the (social) network in which they are embedded; and [3] individuals often consider separate interactions as independent (even when they are not). ${ }^{21}$ Lemma 2 (and to some extent Theorem 2) offers a connection between these three observations: if the underlying process of the network formation is sufficiently stochastic, and if individuals cannot observe perfectly or learn the entire network structure, then in a large network the correct prior of an individual is that her local environment is a random tree. In a random tree separate observations of individual $i$ are asymptotically independent. Notably, this is only a first stab at an important question; the idea that simplified heuristics that people use to adjust for incomplete knowledge of their network can be explained as 'averaging' over a stochastic prior deserves further exploration.

A by-product of Lemma 2 is the provision of sufficient conditions under which a network is expected to exhibits no degree correlation. 'No degree correlation' is often assumed in theoretical models of games in networks (e.g. Jackson and Yariv 2007). Lemma 2 provides a plausible microfoundation. For an application of Lemma 2 in the context of network based hiring in entry level labor markets see also Fainmesser (2010b).

\subsection{Third-party observability, barriers to entry, and efficiency}

Proposition 1 suggests that sufficient WOM and third-party observability allow for a larger set of buyer-seller networks to be cooperation networks. As cooperation increases aggregate welfare in our model, Proposition 1 suggests that facilitating credible communication between buyers is welfare enhancing. Theorem 3 provides a more direct positive result: in the presence of perfect communication channels between buyers, for large $n_{b}$

\footnotetext{
${ }^{21}$ DeMarzo, Vayanos, and Zwiebel (2003) propose a model in which individuals learn from their neighbors about the state of the world. In their model, individuals experience persuasion bias - each individual $i$ continuously updates her prior based on her neighbors' opinions ignoring the fact that her neighbors' opinions depend on the network structure and on information that was previous accessible to $i$. Golub and Jackson (2010) develop a similar model that allows for more flexibility in the updating rule, but maintains the assumption that an individual updates her prior ignoring the network structure.
} 
and $n_{s}$, networks that maximize the volume of trade and networks that maximize the incentives to cooperate are approximately identical. In such 'optimal' networks, the degrees of buyers and sellers are large.

However, Theorem 3 and Corollary 2 also suggest that there are some non-degenerate scenarios in which even with perfect third-party observability there is no network $N=$ $(G, R)$ such that: [1] all sellers in $S$ have an opportunity to trade, and [2] $N$ is a cooperation network. This observation carries welfare implications. We now provide an illustrating example and thereafter discuss the implications of Theorem 3 in the context of the example.

Example 1 Consider $c, \pi, \mu, \delta, n_{b}$, and $n_{s}$ such that $\frac{n_{b}}{n_{s}}<\mu$,

$$
\frac{\delta(\pi-c)}{1-\delta} \cdot \frac{n_{b}}{n_{s}}-c<0,
$$

and

$$
\frac{\delta(\pi-c)}{1-\delta} \cdot \mu-c>0
$$

By Theorem 3, condition 9 guarantees that no network $N$ in which $\operatorname{Pr}\left(d_{s}=0 \mid N\right)=0$ can be a cooperation network. On the other hand, condition 10 assures us that there exists a non-empty cooperation network. For example, a network in which $\operatorname{Pr}\left(d_{b}=1 \mid N\right)=1$, $\operatorname{Pr}\left(d_{s}=1 \mid N\right)=\frac{n_{b}}{n_{s}}$, and $\operatorname{Pr}\left(d_{s}=0 \mid N\right)=1-\frac{n_{b}}{n_{s}}$ is a cooperation network.

In example 1, some sellers are excluded permanently from the market in any cooperation network. Depending on the (unmodeled) network formation mechanism in a given market, this observation lends itself to several interpretations: [1] the need to sustain cooperation may create a barrier to entry, or [2] the existence of barriers to entry may be necessary to facilitate cooperation in some markets.

In an environment in which $\mu<1$, for any finite $n_{s}$ and $n_{b}$, the exclusion of sellers from the market lowers the maximal volume of trade that can be achieved with $n_{s}$ sellers and $n_{b}$ buyers. To see why, note that even if $n_{s}>n_{b}$, in periods in which more than $n_{b}$ sellers produce, the volume of trade is at most $n_{b}$ whereas if less than $n_{b}$ of the connected sellers produce, the volume of trade is bounded above by the number of the connected sellers that produce. 
Nevertheless, in large networks this problem is eliminated. As long as condition (10) holds, there exists an (asymptotically) welfare maximizing cooperation network. We use example 1 to demonstrate this latter point which is an immediate implication of Theorem 3.

Example 1 (cont.) We now focus on the special case where there exist positive integers $d_{B}$ and $d_{S}$, and $\phi_{s} \in(0,1)$ s.t. $\operatorname{Pr}\left(d_{s}=d_{S} \mid \mathcal{D}_{G F}(\cdot)\right)=\phi_{s}, \operatorname{Pr}\left(d_{s}=0 \mid \mathcal{D}_{G F}(\cdot)\right)=1-\phi_{s}$, and $\operatorname{Pr}\left(d_{b}=d_{B} \mid \mathcal{D}_{G F}(\cdot)\right)=1$. Let $\tilde{n_{s}}$ be the number of sellers who have degree $d_{S}$. By construction, $\widetilde{n_{s}}=\phi_{s} \cdot n_{s}=\frac{n_{b} \cdot d_{B}}{d_{S}}$. As long as $\frac{n_{b}}{n_{s}} \geq \mu$, condition 10 implies that $\frac{\delta(\pi-c)}{1-\delta} \cdot \frac{n_{b}}{n_{s}}-c>0$.

Consider $\phi_{s}^{*}$ such that $\tilde{n_{s}}=\frac{n_{b}}{\mu}$. Note that fixing $\phi_{s}$ implies a fixed ratio $\frac{d_{B}}{d_{S}}$. Then by Theorem 3, $\lim _{d_{B} \rightarrow \infty} \lim _{n_{b} \rightarrow \infty}\left(\frac{E\left[V\left(\mathcal{D}_{G F}\left(n_{b}\right)\right)\right]}{n_{b}} \mid \phi_{s}^{*}\right)=1$, and

$$
\lim _{d_{B} \rightarrow \infty} \lim _{n_{b} \rightarrow \infty}\left(I C\left(\mathcal{D}_{G F}\left(n_{b}\right)\right)-\left[\frac{\delta(\pi-c)}{1-\delta} \cdot \mu-c\right] \mid \phi_{s}^{*}\right) \geq 0
$$

which guarantees that given large enough $n_{b}$ and $n_{s}$, there exists a cooperation network that facilitates the maximal expected volume of trade possible (which equals the number of buyers in the market).

\section{Conclusion}

This paper presents a model of repeated games in buyer-seller networks with a reputation network that allows buyers to share information about past transactions with different sellers. The model allows us to vary separately the cooperation network between buyers and sellers and the reputation network between buyers, and examine how the quality of the reputation network affects the ability to sustain cooperation in any given buyer-seller network.

More broadly, our contributions are two-fold, making both a new methodological contribution in the form of a novel method for moving beyond the assumption that a player's payoff function depends only on the actions taken by her immediate neighbors, and an applied contribution in the form of a study of repeated interactions and community enforcement in networked markets with buyers and sellers. 
At the core of our methodological contribution is a new method for reducing questions about the global properties of a networked market to questions about the local properties of the network. This allows us to analyze large networks as if they were small. Our methodology can be applied to various economically important network interaction as long as the effect of one economic agent on another is a decreasing function of the network distance between the two agents on the equilibrium path.

The introduction of incomplete knowledge of the network structure allows us to approximate any small neighborhood of the network with a simple representative network - a random tree. This contribution simplifies the analysis of any environment for which we can reduce questions about the global properties the network to questions about the local properties of the network. Both Theorem 1 and Lemma 2 can be modified to include networks that are not bipartite; allowing for a richer set of interactions.

By applying our methodology to the study of repeated games in networks, we show that while good reputation networks allow for cooperation in markets that could not sustain cooperation otherwise, they do not guarantee cooperation in every market. Surprisingly, a market with high quality reputation networks between buyers can exhibit barriers-to-entry because the number of sellers that can be a part of any cooperation network is a bounded function of the number of buyers in the market. The exclusion of sellers from the market can hinder efficiency in any small market. However, as a market grows, a high quality reputation network that allows for optimal welfare emerges. Such network facilitates the maximal volume of trade as well as sustains cooperation between every seller and buyer that are connected.

\section{Appendix A: proof of Lemma 2}

Lemma 2 has implications that go beyond its role in the analysis of repeated games in networks. For example, Fainmesser (2010b) employs a variant of the Lemma for simplifying the analysis of networked labor markets in a static setting. Results of a similar flavor have also been found useful in other disciplines. ${ }^{22}$ To this end, we present the proof of Lemma 2 as a stand-alone section and follow the conventions of the graph theoretic literature with respect to notation and definitions. We hope that this will make it easier for our more technical readers to appreciate the generality of the result and to

\footnotetext{
${ }^{22}$ See Richardson and Urbanke (2008) for an example from coding theory.
} 
be able to adopt the result or parts of it to be used in further applications.

\subsection{Notations and definitions}

A graph $\Gamma=(V, E)$ is a set of nodes $V$ and a set of edges $E$, where each edge $e=\left(v_{1}, v_{2}\right)$ specifies that there is a connection between nodes $v_{1}$ and $v_{2}$. To prove Lemma 2 we introduce a particular randomization scheme (which we will soon describe in depth). We first formalize the class of graphs over which we randomize, and the different notions of degree distribution (d.d.) that we will use. A graph $\Gamma$ is bipartite if and only if $\Gamma$ can be partitioned into two sets (e.g. S(ellers) and B(uyers)) such that all edges contain exactly one node from $S$ and one node from $B$. A bipartite graph is said to be bicolored if the nodes of the one partite are distinguished from the nodes of the other partite. For example, the bicolored property guarantees that the graph on three nodes in which one seller node is connected to two buyer nodes is distinguished from the graph on three nodes in which one buyer node is connected to two seller nodes. We say that a bicolored bipartite graph is labeled if each node in partite $S$ have a distinct label from the set $\left\{1, \ldots, n_{s}\right\}$, and each node in partite $B$ have a distinct label from the set $\left\{1, \ldots, n_{b}\right\}$. A graph $\Gamma$ is rooted if one of the nodes on $\Gamma$ is labelled in a special way to distinguish it from the graph's other nodes. This special node is called the root of the graph. For two rooted graphs $\Gamma_{1}, \Gamma_{2}$, we say that $\Gamma_{1}=\Gamma_{2}$ if the two graphs are isomorphic with respect to the root. For a node $v$ in a graph $\Gamma$, recall that $d_{v}$ denotes the degree (number of neighbors) of $v$ in $\Gamma$. Sometimes to make the underlying graph explicit, we use the notation $d_{v}^{\Gamma}$.

For a graph $\Gamma$ and a subset of nodes $V^{\prime}$ of $\Gamma$, the subgraph induced by $V^{\prime}$ will refer to the subgraph of $\Gamma$ consisting of the nodes $V^{\prime}$ and all edges in $\Gamma$ that connect nodes in $V^{\prime}$. Recall that for a given node $v$ and depth $d, N_{d}(v)$ was earlier defined as the set of nodes whose graphical distance from $v$ is at most $d$. For the remainder of Appendix A, $N_{d}(v)$ should be read as referring not just to the given set of nodes, but the subgraph induced by that set of nodes. Sometimes, to make the reference graph explicit, we use the notation $N_{d}^{\Gamma}(v)$. Also, for a given node $v$ in a graph $G$, we let $F_{G}(v)$ denote the set of degrees of the nodes adjacent to $v$ in $G$. Recall that the set of degrees of a given bipartite graph $\Gamma$ may be defined in two distinct ways. Let $\mathcal{H}_{\Gamma}^{s}\left(\mathcal{H}_{\Gamma}^{b}\right)$ denote the random variable (r.v.) representing the degree of a seller (buyer) node selected u.a.r. from all seller (buyer) nodes. Alternatively, let $\mathcal{G}_{\Gamma}^{s}\left(\mathcal{G}_{\Gamma}^{b}\right)$ denote the r.v. representing the degree of the seller (buyer) belonging to an edge selected u.a.r. from all edges of $\Gamma$.

For concreteness, let us fix some given degree distributions $\mathcal{H}^{s}, \mathcal{H}^{b}$ with finite, nonnegative support and rational probabilities. We let $m_{\mathcal{H}}$ denote some integer bound on the support of both $\mathcal{H}^{s}$ and $\mathcal{H}^{b}$. Let $\mathcal{G}^{s}, \mathcal{G}^{b}$ denote the corresponding degree distributions under the random edge interpretation. Let $\mathcal{G}\left(n_{b}\right)$ denote the set of labeled bicolored bipartite graphs that satisfy d.d. $\mathcal{G}^{s}, \mathcal{G}^{b}$, and in which the buyer partite has $n_{b}$ nodes. We let $n_{s}$ denote the corresponding number of nodes in the seller partite (determined uniquely by $n_{b}$ and $\left.\mathcal{G}^{s}, \mathcal{G}^{b}\right)$. Let $\mathcal{R}\left(n_{b}\right)$ denote a graph selected u.a.r. from $\mathcal{G}\left(n_{b}\right)$. Let $\mathcal{R}^{S}\left(n_{b}\right)\left(\mathcal{R}^{B}\left(n_{b}\right)\right)$ denote the set of nodes in the seller (buyer) partite of $\mathcal{R}\left(n_{b}\right)$. Let $\mathcal{F}$ denote the set of vectors $\mathbf{f}$ s.t. $\operatorname{Pr}\left(F_{\mathcal{R}\left(n_{b}\right)}(v)=\mathbf{f}\right)>0$ for some $v \in \mathcal{R}\left(n_{b}\right)$ (note that $\mathcal{F}$ 
is dictated by $\left.\mathcal{H}^{s}, \mathcal{H}^{b}, n_{b}\right)$.

Note that the random graph $\mathcal{R}\left(n_{b}\right)$ has some non-trivial dependencies. Indeed, if one conditions on there being an edge between nodes $s$ and $b$, the precise effect of this conditioning on the degrees of the other nodes is difficult to characterize exactly; large-scale dependencies are introduced by the condition that the graph has the global structure dictated by $\mathcal{H}^{s}, \mathcal{H}^{b}$. In spite of this, we prove that the local structure of $\mathcal{R}\left(n_{b}\right)$ is quite simple, namely that of a tree in which the degrees are chosen i.i.d. Let $\mathcal{T}(d, r)$ denote a rooted depth- $d$ tree generated as follows. The degree of the root equals $r$. Each node at an even depth $k \leq d-1$ is given an i.i.d. number of children distributed as $\mathcal{G}^{b}-1$, and each node at odd depth $k \leq d-1$ is given an i.i.d. number of children distributed as $\mathcal{G}^{s}-1$.

Note that to prove Lemma 2, it suffices to show the following.

Lemma 4 For all $\mathbf{f} \in \mathcal{F}$ and trees $T$,

$\lim _{n_{b} \rightarrow \infty} \sup _{v \in \mathcal{R}^{S}\left(n_{b}\right)}\left|\operatorname{Pr}\left(N_{d}^{\mathcal{R}\left(n_{b}\right)}(v)=T \mid F_{\mathcal{R}\left(n_{b}\right)}(v)=\mathbf{f}\right)-\operatorname{Pr}\left(\mathcal{T}\left(d, d_{v}\right)=T \mid F_{\mathcal{T}\left(d, d_{v}\right)}(v)=\mathbf{f}\right)\right|=0$.

\subsection{Configuration method}

To analyze $\mathcal{R}\left(n_{b}\right)$ and prove Lemma 2 , it will be convenient to analyze the well-known pairing (a.k.a. configuration) method for generating $\mathcal{R}\left(n_{b}\right)$ (see e.g. Greenhill et al. 2006, Section 2). First, construct $n_{s}$ seller buckets $S_{1}, S_{2}, \ldots, S_{n_{s}}$ and $n_{b}$ buyer buckets $B_{1}, B_{2}, \ldots, B_{n_{b}}$. Second, for each $d \geq 1$, populate a $\operatorname{Pr}\left(\mathcal{H}^{s}=d\right)\left(\operatorname{Pr}\left(\mathcal{H}^{b}=d\right)\right.$ fraction of seller (buyer) buckets with exactly $d$ indistinguishable points. Here we let $\left|S_{i}\right|\left(\left|B_{j}\right|\right)$ denote the number of points assigned to bucket $S_{i}\left(B_{j}\right)$, and $n_{b, p}\left(n_{s, p}\right)$ denote the total number of buyer (seller) points as dictated by $n_{b}, \mathcal{H}^{s}$, and $\mathcal{H}^{b}$. Third, select a matching $\mathcal{M}\left(n_{b}\right)$ between the seller points and the buyer points u.a.r. Fourth, construct a labeled bicolored bipartite graph $R^{\prime}\left(n_{b}\right)$ such that there are $n_{b}$ buyer nodes, $n_{s}$ seller nodes, and an edge connecting seller node $s_{i}$ and buyer node $b_{j}$ iff at least one point belonging to seller bucket $S_{i}$ was matched to a point belonging to buyer bucket $B_{j}$. Note that it is possible that in $\mathcal{M}\left(n_{b}\right)$, there exist buckets $S_{i}, B_{j}$ such that two points in $S_{i}$ are connected to two points in $B_{j}$, in which case the d.d. of $\mathcal{R}^{\prime}\left(n_{b}\right)$ need not be the same as that of $\mathcal{R}\left(n_{b}\right)$.

Our approach to proving Lemma 4 will be to first prove an analogue (but without the conditioning involving $\mathcal{F}$ ) for $\mathcal{R}^{\prime}\left(n_{b}\right)$.

Lemma 5 For all trees $T, \lim _{n_{b} \rightarrow \infty} \sup _{v \in \mathcal{R}^{\prime S}\left(n_{b}\right)} \mid \operatorname{Pr}\left(N_{d}^{\mathcal{R}^{\prime}\left(n_{b}\right)}(v)=T\right)-\operatorname{Pr}\left(\mathcal{T}\left(d, d_{v}\right)=\right.$ $T) \mid=0$.

Proof. Note that we may construct the random matching $\mathcal{M}\left(n_{b}\right)$ in the following manner. First, we pick an arbitrary seller or buyer point $p_{1}$ of our choice. Then, if $p_{1}$ was a seller point, we select a point $p_{2}$ u.a.r. from all buyer points. Alternatively, if $p_{1}$ was a buyer point, we select a point $p_{2}$ u.a.r. from all seller points. We then add edge $\left(p_{1}, p_{2}\right)$ to $\mathcal{M}\left(n_{b}\right)$; eliminate $p_{1}$ and $p_{2}$ from the set of remaining points; and repeat until all points are matched. It follows that we may construct $\mathcal{M}\left(n_{b}\right)$ by selecting the points in an order 
such that for any bucket $S_{i}$ of our choosing, $N_{d}\left(s_{i}\right)$ is 'generated first'. Roughly speaking, we first pair off those points whose buckets will eventually correspond to neighbors of a seller $s_{i}$ in $\mathcal{R}^{\prime}\left(n_{b}\right)$; we then pair off those points whose buckets will eventually become neighbors of neighbors of $s_{i}$ in $\mathcal{R}^{\prime}\left(n_{b}\right)$, etc. More precisely, we may construct the matching $\mathcal{M}\left(n_{b}\right)$ using the following algorithm. We proceed through a series of stages, indexed by $k$. We will decide which point we pair off next (more precisely the bucket containing that point) by assigning the buckets labels as the algorithm proceeds.

\section{RANDGEN:}

Initialize: $k=1$. Assign bucket $S_{i}$ the label 1 .

While there exists at least one unmatched point:

While there exists at least one bucket with label $k$ :

Select a bucket $U$ u.a.r. from all buckets with label $k$ :

Select an unmatched point $p$ u.a.r. from $U$ :

Select an unmatched point $p^{\prime}$ u.a.r. from all unmatched seller (buyer) points; Add edge $\left(p, p^{\prime}\right)$ to $\mathcal{M}\left(n_{b}\right)$;

Remove points $p, p^{\prime}$ from the set of remaining points;

Assign the bucket containing point $p^{\prime}$ the label $k+1$;

If there does not exist a bucket with label $k+1$ containing at least one unmatched point:

Select a bucket $U$ u.a.r. from all seller(buyer) buckets with $\geq 1$ unmatched point;

Assign bucket $U$ label $k+1$;

$k=k+1$;

A simple proof by contradiction shows that $R A N D G E N$ always terminates, and a simple induction shows that no bucket is ever assigned two different labels. Note that since each time we pick a point we match it u.a.r. to a remaining point of the 'other' partite, $R A N D G E N$ indeed returns a matching distributed u.a.r.

Let $E_{i, \Delta}$ be the event that no bucket with label $k \leq \Delta+1$ was assigned its label more than once.

Observation 1 Conditional on the event $E_{i, \Delta}, N_{\Delta}^{\mathcal{R}^{\prime}\left(n_{b}\right)}\left(s_{i}\right)$ is acyclic.

By a simple induction, at most $2\left(m_{\mathcal{H}}+m_{\mathcal{H}}\left(m_{\mathcal{H}}-1\right)+m_{\mathcal{H}}\left(m_{\mathcal{H}}-1\right)^{2}+\ldots+m_{\mathcal{H}}\left(m_{\mathcal{H}}-\right.\right.$ $\left.1)^{\Delta-1}\right) \leq 2 \Delta m_{\mathcal{H}}^{\Delta}$ points are matched while $k \leq \Delta$. Let $p_{1}, p_{2}$ be any two points belonging to sellers' buckets matched during stage $k \leq \Delta$ for $k$ even. Then the probability that $p_{1}, p_{2}$ were matched to points $q_{1}, q_{2}$ belonging to the same buyer's bucket is at most $\frac{m_{\mathcal{H}}-1}{n_{b, p}-2 \Delta m_{\mathcal{H}}^{\Delta}}$. Indeed, w.l.o.g. assuming $p_{1}$ was matched first (with $q_{1}$ ), there are at most $m_{\mathcal{H}}-1$ points out of at least $n_{b, p}-2 \Delta m_{\mathcal{H}}^{\Delta}$ remaining points which $q_{2}$ could be matched to so that $q_{1}, q_{2}$ belong to the same bucket. Since there are at most $\left(\begin{array}{c}2 \Delta m_{\mathcal{H}} \\ 2\end{array}\right)$ pairs of points such that both are matched during stage $k \leq \Delta$, it follows from a union bound that

$$
\operatorname{Pr}\left(E_{i, \Delta}\right) \geq 1-\left(\begin{array}{c}
2 \Delta m_{\mathcal{H}}^{\Delta} \\
2
\end{array}\right) \frac{m_{\mathcal{H}}-1}{n_{b, p}-2 \Delta m_{\mathcal{H}}^{\Delta}}=1-O\left(\frac{1}{n_{b}}\right) .
$$


Let $U$ be any seller bucket assigned label $k \leq \Delta$, and $p$ any point in $U$ that is matched during stage $k$. It follows from (11) and the previous discussion that for any $i$, regardless of the value of $d_{s_{i}}$ and the actions taken by RANDGEN before $p$ was matched, the probability that $p$ is matched to a point $q$ contained in a bucket $B_{i}$ satisfying $d_{b_{i}}^{\mathcal{R}^{\prime}\left(n_{b}\right)}=l$ is at least $\operatorname{Pr}\left(\mathcal{H}^{b}=l\right)-O\left(\frac{1}{n_{b}}\right)$. Similarly, the probability that $p$ is matched to a point

$q$ contained in a bucket $B_{i}$ satisfying $d_{b_{i}}^{\mathcal{R}^{\prime}\left(n_{b}\right)}=l$ is at most $\operatorname{Pr}\left(\mathcal{H}^{b}=l\right)+O\left(\frac{1}{n_{b}}\right)$. We note that corresponding bounds hold with the role of buyers and sellers interchanged. It follows that the number of points in the bucket chosen next by $R A N D G E N$ is asymptotically independent and identically distributed, where the associated distributions (which depend only on whether the current bucket is a buyer or seller bucket) correspond to $\mathcal{H}^{s}, \mathcal{H}^{b}$. Lemma 5 then follows from a standard coupling argument, in which we construct $\mathcal{T}\left(d, d_{v}\right)$ and $N_{\mathcal{R}^{\prime}\left(n_{b}\right)}^{d}(v)$ on the same probability space.

\subsection{Relating the configuration model back to the original model}

We now relate $\mathcal{R}\left(n_{b}\right)$ to $\mathcal{R}^{\prime}\left(n_{b}\right)$ probabilistically. Namely, it is well-known (see e.g. Greenhill et al. 2006) that

Lemma $6 \mathcal{R}\left(n_{b}\right)$ is distributed exactly as $\mathcal{R}^{\prime}\left(n_{b}\right)$ conditioned to belong to the set $\mathcal{G}\left(n_{b}\right)$.

We now bound the probability that $\mathcal{R}^{\prime}\left(n_{b}\right)$ belongs to $\mathcal{G}\left(n_{b}\right)$. In particular, it follows from Theorem 1.3 and Lemma 2.1 of Greenhill et. al. 2006 that for the fixed degree distributions $\mathcal{H}^{s}, \mathcal{H}^{b}$

Lemma $7 \lim _{n_{b} \rightarrow \infty} \operatorname{Pr}\left(\mathcal{R}^{\prime}\left(n_{b}\right) \in \mathcal{G}\left(n_{b}\right)\right)>0$.

\subsection{Completing the proof of Lemma 4}

The only remaining hurdle to proving Lemma 4 is to 'reincorporate' the conditioning involving $\mathcal{F}$. This can be proven directly by computing the relevant conditional probabilities. However, we offer an alternative proof that is more general. We show that for almost all graphs in $\mathcal{G}\left(n_{b}\right)$, the fraction of nodes whose neighborhood is isomorphic to any given tree $T$ is approximately the same as the probability that a corresponding i.i.d. random tree is isomorphic to $T$. Therefore, the fact that a seller knows her degree and the degrees of buyers connected to her does not affect the seller's posterior over the global network structure, or even over her local network structure that is not included in her explicit knowledge. We do that by proving a concentration result, namely that for any tree $T$, the variance of the number of sellers whose neighborhood looks like $T$ in $\mathcal{R}^{\prime}\left(n_{b}\right)$ goes to zero as $n_{b}$ goes to infinity.

Lemma 8 For any rooted tree $T, \operatorname{Var}\left[n_{s}^{-1} \sum_{s_{i}} I\left(N_{\Delta}^{\mathcal{R}^{\prime}\left(n_{b}\right)}\left(s_{i}\right)=T\right)\right]=O\left(\frac{1}{n_{s}}\right)$.

Proof. After expanding the variance using its definition as the difference between the expected value of the square and the square of the expectation, the only non-trivial step in proving Lemma 8 is bounding the covariance of the indicators $I\left(N_{\Delta}^{\mathcal{R}^{\prime}\left(n_{b}\right)}\left(s_{i}\right)=\right.$ 
$T), I\left(N_{\Delta}^{\mathcal{R}^{\prime}\left(n_{b}\right)}\left(s_{j}\right)=T\right)$ for (arbitrary) nodes $s_{i}, s_{j}$. To analyze this covariance, we consider implementing RANDGEN in a slightly modified manner- namely, we generate 'both' $N_{\Delta}^{\mathcal{R}^{\prime}\left(n_{b}\right)}\left(s_{i}\right)$ and $N_{\Delta}^{\mathcal{R}^{\prime}\left(n_{b}\right)}\left(s_{j}\right)$ 'first'. More precisely, let $R A N D G E N^{\prime}$ be the algorithm that is equivalent to $R A N D G E N$, except at initialization both buckets $S_{i}$ and $S_{j}$ are assigned the label 1. The covariance of $I\left(N_{\Delta}^{\mathcal{R}^{\prime}\left(n_{b}\right)}\left(s_{i}\right)=T\right), I\left(N_{\Delta}^{\mathcal{R}^{\prime}\left(n_{b}\right)}\left(s_{j}\right)=T\right)$ is then bounded by analyzing RANDGEN $N^{\prime}$ to show that $N_{\Delta}^{\mathcal{R}^{\prime}\left(n_{b}\right)}\left(s_{i}\right)$ and $N_{\Delta}^{\mathcal{R}^{\prime}\left(n_{b}\right)}\left(s_{j}\right)$ are asymptotically independent (in an appropriate sense). The analysis proceeds very similarly to our proof of (11), and we omit the details.

\section{Appendix B: additional proofs}

Theorem 3 - Proof. As all sellers are symmetric, we have that $E\left[V\left(\mathcal{D}_{G F}\left(R^{1}, n_{b}, d_{S}, d_{B}\right)\right)\right]=n_{s} \sum_{b \in N_{1}(s)} \operatorname{Pr}(I(s, b)=1)$, where $s$ is any seller. Furthermore, as only those sellers which produce can trade, we have the further refinement

$$
E\left[V\left(\mathcal{D}_{G F}\left(R^{1}, n_{b}, d_{S}, d_{B}\right)\right)\right]=\mu n_{s} \sum_{b \in N_{1}(s)} \operatorname{Pr}(I(s, b)=1 \mid s \text { produces }) .
$$

Let $T^{1}\left(d, d_{S}, d_{B}\right)$ denote the rooted depth- $d$ tree (with root $\left.r^{1}\right)$ s.t. the root has $d_{S}$ children, each non-leaf node at odd depth has $d_{B}-1$ children, and each non-leaf node at even depth has $d_{S}-1$ children. For $0<\mu<1$, let $T^{1}\left(d, d_{S}, d_{B}, \mu\right)$ denote the random rooted depth- $d$ tree (with root $\left.r^{1}\right)$ constructed by taking $T^{1}\left(d, d_{S}, d_{B}\right)$ and deleting each seller (other than $r^{1}$ ) w.p. $\mu$ (i.i.d. across sellers). For a graph $G$, let $\mathcal{M}(G)$ denote the random greedy graph matching (on $G$ ) constructed by examining the edges of $G$ in a u.a.r. permutation, always including an edge iff no incident edge has already been included (the same studied in GG). For a node $v \in G$, let $I(v \in \mathcal{M}(G))$ denote the indicator for the event that $v$ is matched in $\mathcal{G}$ (equivalently $v$ is incident to a selected edge). Then it follows from Lemma 2, Lemma 6 of GG, and (12) that for any $\epsilon>0$, there exists $N_{\epsilon, d_{S}, d_{B}, \mu}, d_{\epsilon, d_{S}, d_{B}, \mu}$ (depending only on $\epsilon, d_{S}, d_{B}, \mu$ ) s.t. for all $n_{s}, n_{b} \geq N_{\epsilon, d_{S}, d_{B}, \mu}$ and $d \geq d_{\epsilon, d_{S}, d_{B}, \mu}$,

$$
\left|\frac{E\left[V\left(\mathcal{D}_{G F}\left(R^{1}, n_{b}, d_{S}, d_{B}\right)\right)\right]}{n_{s}}-\mu \operatorname{Pr}\left(r_{1} \in \mathcal{M}\left(T^{1}\left(d, d_{S}, d_{B}, \mu\right)\right)\right)\right|<\epsilon,
$$

and thus

$$
\frac{E\left[V\left(\mathcal{D}_{G F}\left(R^{1}, n_{b}, d_{S}, d_{B}\right)\right)\right]}{n_{s}} \geq \mu \operatorname{Pr}\left(r_{1} \in \mathcal{M}\left(T^{1}\left(d, d_{S}, d_{B}, \mu\right)\right)\right)-\epsilon .
$$

It follows from Lemma 3 that $\operatorname{Pr}\left(r_{1} \in \mathcal{M}\left(T^{1}\left(d, d_{S}, d_{B}, \mu\right)\right)\right) \geq \operatorname{Pr}\left(r_{1} \in \mathcal{M}\left(T^{1}\left(d, d_{S}, d_{B}\right)\right)\right)$, since deleting sellers is equivalent to removing (as children) subtrees from buyer nodes. 
Combining with (14), we find that for all $n_{s}, n_{b} \geq N_{\epsilon, d_{S}, d_{B}, \mu}$ and $d \geq d_{\epsilon, d_{S}, d_{B}, \mu}$

$$
\frac{E\left[V\left(\mathcal{D}_{G F}\left(R^{1}, n_{b}, d_{S}, d_{B}\right)\right)\right]}{n_{s}} \geq \mu \operatorname{Pr}\left(r_{1} \in \mathcal{M}\left(T^{1}\left(d, d_{S}, d_{B}\right)\right)\right)-\epsilon .
$$

We now treat two cases. First, suppose $d_{S} \geq d_{B}$. Then it follows from Lemma 3 that

$$
\operatorname{Pr}\left(r_{1} \in \mathcal{M}\left(T^{1}\left(d, d_{S}, d_{B}\right)\right)\right) \geq \operatorname{Pr}\left(r_{1} \in \mathcal{M}\left(T^{1}\left(d, d_{S}, d_{S}\right)\right)\right) .
$$

But it follows from Corollary 6 of GG (in light of Lemma 6 of GG) that for all $d_{S} \geq 3$,

$$
\lim _{d \rightarrow \infty} \operatorname{Pr}\left(r_{1} \in \mathcal{M}\left(T^{1}\left(d, d_{S}, d_{S}\right)\right)\right)=1-\left(d_{S}-1\right)^{-\frac{d_{S}}{d_{S}-2}} .
$$

Thus since $\frac{d_{S}}{d_{S}-2} \geq 1$, we have that

$$
\lim _{d \rightarrow \infty} \operatorname{Pr}\left(r_{1} \in \mathcal{M}\left(T^{1}\left(d, d_{S}, d_{S}\right)\right)\right) \geq 1-\frac{1}{d_{S}-1} .
$$

Combining (15),(16), and (18) demonstrates the Theorem for the case $d_{S} \geq d_{B}$.

Now, suppose $d_{B} \geq d_{S}$. It follows from Lemma 2 that for any fixed $d, \epsilon, d_{B}, d_{S}$, there exists a bipartite graph $G\left(d, \epsilon, d_{B}, d_{S}\right)$ (with partites $\left.B, S\right)$ s.t.: 1 . all nodes in partite $B$ have degree $d_{B}$ and all nodes in partite $S$ have degree $d_{S}$, and 2. a $1-\epsilon$ fraction of nodes in partite $S$ (partite $B$ ) have depth- $d$ neighborhoods isomorphic to $T^{1}\left(d, d_{S}, d_{B}\right)\left(T^{1}\left(d, d_{B}, d_{S}\right)\right)$. By Lemma 6 of GG, for any fixed $\epsilon, d_{B}, d_{S}$ we may select a sufficiently large $d \triangleq d\left(\epsilon, d_{B}, d_{S}\right)$ s.t. for any node $s$ belonging to the (at least) $(1-\epsilon)|S|$ nodes of partite $S$ with depth- $d$ neighborhoods isomorphic to $T^{1}\left(d, d_{S}, d_{B}\right), \mid \operatorname{Pr}(s \in$ $\left.\mathcal{M}\left(G\left(d, \epsilon, d_{B}, d_{S}\right)\right)\right)-\operatorname{Pr}\left(r_{1} \in \mathcal{M}\left(T^{1}\left(d, d_{S}, d_{B}\right)\right) \mid<\epsilon\right.$. Also, for any node $b$ belonging to the (at least) $(1-\epsilon)|B|$ nodes of partite $B$ with depth- $d$ neighborhoods isomorphic to $T^{1}\left(d, d_{B}, d_{S}\right), \mid \operatorname{Pr}\left(b \in \mathcal{M}\left(G\left(d, \epsilon, d_{B}, d_{S}\right)\right)\right)-\operatorname{Pr}\left(r_{1} \in \mathcal{M}\left(T^{1}\left(d, d_{B}, d_{S}\right)\right) \mid<\epsilon\right.$. Combining the above, we find that for the graph $G\left(d, \epsilon, d_{B}, d_{S}\right)$,

$$
\left|E\left[\sum_{b \in B} I\left(b \in \mathcal{M}\left(G\left(d, \epsilon, d_{B}, d_{S}\right)\right)\right)\right]-\right| B\left|\operatorname{Pr}\left(r_{1} \in \mathcal{M}\left(T^{1}\left(d, d_{B}, d_{S}\right)\right)\right)\right| \leq 2 \epsilon|B|,
$$

and

$$
\left|E\left[\sum_{s \in S} I\left(s \in \mathcal{M}\left(G\left(d, \epsilon, d_{B}, d_{S}\right)\right)\right)\right]-\right| S\left|\operatorname{Pr}\left(r_{1} \in \mathcal{M}\left(T^{1}\left(d, d_{S}, d_{B}\right)\right)\right)\right| \leq 2 \epsilon|S| .
$$


Note that since the number of matched nodes in partite $S$ always equals the number of matched nodes in partite $B$, one has $E\left[\sum_{b \in B} I\left(b \in \mathcal{M}\left(G\left(d, \epsilon, d_{B}, d_{S}\right)\right)\right)\right]=$ $E\left[\sum_{s \in S} I\left(s \in \mathcal{M}\left(G\left(d, \epsilon, d_{B}, d_{S}\right)\right)\right)\right]$. It thus follows from (19) and (20) that

$$
\left|\operatorname{Pr}\left(r_{1} \in \mathcal{M}\left(T^{1}\left(d, d_{B}, d_{S}\right)\right)\right)-\frac{d_{B}}{d_{S}} \operatorname{Pr}\left(r_{1} \in \mathcal{M}\left(T^{1}\left(d, d_{S}, d_{B}\right)\right)\right)\right| \leq 2 \epsilon\left(1+\frac{d_{B}}{d_{S}}\right),
$$

and

$$
\lim _{d \rightarrow \infty} \operatorname{Pr}\left(r_{1} \in \mathcal{M}\left(T^{1}\left(d, d_{B}, d_{S}\right)\right)\right)=\frac{d_{B}}{d_{S}} \lim _{d \rightarrow \infty} \operatorname{Pr}\left(r_{1} \in \mathcal{M}\left(T^{1}\left(d, d_{S}, d_{B}\right)\right)\right) .
$$

Combining with (15), we find that for any fixed $\epsilon, d_{S}, d_{B}, \mu$ there exist $N_{\epsilon, d_{S}, d_{B}, \mu}^{\prime}, d_{\epsilon, d_{S}, d_{B}, \mu}^{\prime}$ s.t. for all $n_{s}, n_{b} \geq N_{\epsilon, d_{S}, d_{B}, \mu}^{\prime}, d \geq d_{\epsilon, d_{S}, d_{B}, \mu}^{\prime}$,

$$
\frac{E\left[V\left(\mathcal{D}_{G F}\left(R^{1}, n_{b}, d_{S}, d_{B}\right)\right)\right]}{n_{s}} \geq \mu \frac{d_{S}}{d_{B}} \operatorname{Pr}\left(r_{1} \in \mathcal{M}\left(T^{1}\left(d, d_{B}, d_{S}\right)\right)\right)-\epsilon .
$$

It follows from Lemma 3 that $\operatorname{Pr}\left(r_{1} \in \mathcal{M}\left(T^{1}\left(d, d_{B}, d_{S}\right)\right)\right) \geq \operatorname{Pr}\left(r_{1} \in \mathcal{M}\left(T^{1}\left(d, d_{B}, d_{B}\right)\right)\right)$. Combining with (18) (replacing $d_{S}$ by $d_{B}$ ) and taking limits demonstrates the theorem for the case $d_{B} \geq d_{S}$.

Corollary 2 - Proof. Part 1: Let $\mu=1$ and $\gamma \geq 1$. Let $N=(G, R)$ be any network that satisfy the following: [1] for every $b \in B, d_{b}=1$; and [2] $\max \left\{d_{s}\right\}_{s \in S}=$ 1. The network $N$ consists of $\bar{n}_{b}$ buyer-seller pairs and $\gamma \bar{n}_{b}-\bar{n}_{b}$ sellers that are note connected to any buyer ( $R$ can be chosen arbitrarily). Let $\Delta N$ put probability 1 on the network $N$. Then,

$$
E[V(\Delta N)]=\bar{n}_{b} \text { and } \min _{s \in S} I C(c, \pi, \mu, \delta, \Delta N)=\frac{\delta(\pi-c)}{1-\delta} \cdot \mu-c>0 .
$$

Plugging (24) into the definition of $W L\left(\bar{n}_{b}, \bar{n}_{s}, c, \pi, \mu, \delta\right)$ complete the proof. The proof for the case where $\gamma<1$ is symmetric.

Part 2: Assume by contradiction that for every $\bar{\gamma}$ there exists $\gamma>\bar{\gamma}$ such that $W L\left(\bar{n}_{b}, \gamma \bar{n}_{b}, c, \pi, \mu, \delta\right)=0$. Let $\bar{n}_{s}^{t}$ be the number of sellers that are able to produce in period $t$. The contradiction assumption implies that there exists $\Delta N$ such that in every period $\min \left\{\bar{n}_{b}, \bar{n}_{s}^{t}\right\}$ trades take place and that $\min _{s \in S} I C(c, \pi, \mu, \delta, \Delta N)>0$. However, given that $\mu<1$, to satisfy that in every period $\min \left\{\bar{n}_{b}, \bar{n}_{s}^{t}\right\}$ trades take place, $\Delta N$ must provide each seller with a positive probability of selling in every period that she produces. Thus, for $\gamma>\frac{1}{\mu}, \min _{s \in S} I C(c, \pi, \mu, \delta, \Delta N)<\frac{\delta(\pi-c)}{1-\delta} \cdot \frac{1}{\gamma}-c$ which is guaranteed to be negative for any $\gamma>\frac{\delta(\pi-c)}{(1-\delta) c}$. This completes the proof by contradiction to $\min _{s \in S} I C(c, \pi, \mu, \delta, \Delta N)>0$. 
Part 3: Let $\gamma \geq \frac{1}{\mu}$ and $n_{s}=\frac{1}{\mu} \cdot \bar{n}_{b}$. Let $\Delta N$ assign identical probability to any network that is possible conditional on the following: [1] $d_{s}=d^{S}$ for exactly $n_{s}$ sellers and $d_{s}=0$ for $\bar{n}_{s}-n_{s}$ seller; and [2] $d_{b}=d^{B}=\frac{1}{\mu} \cdot d^{S}$ for every $b \in B$. Combining Theorem 3 and Equation (8) we get that

$$
\lim _{d^{S}} \lim _{\bar{n}_{b} \rightarrow \infty} \frac{E[V(\Delta N)]}{\bar{n}_{b}} \geq 1, \text { and } \lim _{d^{S}} \lim _{\bar{n}_{b} \rightarrow \infty} I C(c, \pi, \mu, \delta, \Delta N) \geq \frac{\delta(\pi-c)}{1-\delta} \cdot \mu-c>0,
$$

which completes the proof. The proof for the case where $\gamma<\frac{1}{\mu}$ is much simpler and follows a similar logic and therefore omitted.

\section{References}

Acemoglu, Daron, Munther A. Dahleh, Ilan Lobel, and Asuman Ozdaglar (2010), "Bayesian Learning in Social Networks," working paper.

Ballester, Coralio, Antoni Calvó-Armengol, and Yves Zenou (2006), "Who's Who in Networks. Wanted: The Key Player," Econometrica, 74, 1403-1417.

Banerjee, Abhijit V. and Andrew F. Newman (1993), "Occupational Choice and the Process of Development," Journal of Political Economy, vol. 101(2), pages 274-98.

Barabási, Albert-László and Réka Albert (1999), "Emergence of Scaling in Random Networks," Science, Vol. 286. no. 5439, pp. 509 - 512.

Bayati, Mohsen, David Gamarnik, Dimitriy A. Katz, Chandra Nair, and Prasad Tetali (2007), "Simple Deterministic Approximation Algorithms for Counting Matchings," Proceedings of the thirty-ninth annual ACM symposium on Theory of computing (STOC '07), pp. 122-127.

Blume, Larry, David Easley, Jon Kleinberg, and Eva Tardos (2007), "Trading Networks with Price-Setting Agents," Proc. 8th ACM Conference on Electronic Commerce.

Bolton, Gary E., Ben Greiner, and Axel Ockenfels (2009), "Engineering Trust Reciprocity in the Production of Reputation Information," UNSW Australian School of Business Research, Paper No. 2009 ECON 02.

Bramoullé, Yann, Martin D’Amours, and Rachel Kranton (2010), "Strategic Interaction and Networks," working paper.

Dellarocas, Chrysanthos N. (2000), "Immunizing Online Reputation Reporting Systems Against Unfair Ratings and Discriminatory Behavior," Proceedings of the 2nd ACM Conference on Electronic Commerce, Minneapolis, MN.

Dellarocas, Chrysanthos N. (2001), "Building Trust On-Line: The Design of Reliable Reputation Reporting Mechanisms for Online Trading Communities," MIT Sloan Working Paper No. 4180-01.

DeMarzo, Peter M., Dimitri Vayanos, and Jeffrey Zwiebel (2003), "Persuasion Bias, Social Influence, and Unidimensional Opinions" The Quarterly Journal of Economics, 118, 909-968. 
Ellison, Glenn (1994), "Cooperation in the Prisoner's Dilemma with Anonymous Random Matching," Review of Economic Studies, 61, 567-588.

Ellison, Glenn and Drew Fudenberg (1995), "Word-of-Mouth Communication and Social Learning," The Quarterly Journal of Economics, 110, 93-125.

Esfahani, Hadi S. and Djavad Salehi-Isfahani (1989), "Effort Observability and Worker Productivity: Towards an Explanation of Economic Dualism," Economic Journal, vol. 99(397), pages 818-36.

Fafchamps, Marcel (1996), "The Enforcement of Commercial Contracts in Ghana," World Development, 24, 427-448.

Fainmesser, Itay P. (2010a), "Community Structure and Market Outcomes: A Repeated Games in Networks Approach," working paper.

Fainmesser, Itay P. (2010b), "Social Networks and Unraveling in Labor Markets," working paper.

Gale, Douglas and Shachar Kariv (2003), "Bayesian learning in social networks," Games and Economic Behavior 45, 329-346.

Galeotti, Andrea and Sanjeev Goyal (2009), "A Theory of Strategic Diffusion," Rand Journal of Economics, 40 (3), 509-532.

Galeotti, Andrea, Sanjeev Goyal, Matthew O. Jackson, Fernando Vega-Redondo, and Leeat Yariv (2010), "Network Games," Review of Economic Studies, Vol. 77 (1), pp 218-244.

Gamarnik, David and David Goldberg (2010), "Randomized Greedy Algorithms for Independent Sets and Matchings in Regular Graphs: Exact Results and Finite Girth Corrections," Combinatorics, Probability and Computing, vol. 19, Issue 1, pp.61-85.

Golub, Benjamin and Matthew O. Jackson (2010), "Naive Learning in Social Networks: Convergence, Influence and the Wisdom of Crowds," American Economic Journal: Microeconomics, 2(1), 112-49.

Goring, Frank, Jochen Harant, Dieter Rautenbach, and Ingo Schiermeyer (2009), "Locally Dense Independent Sets in Regular Graphs of Large Girth - An Example of a New Approach," Research Trends in Combinatorial Optimization, pp. 163-183.

Goyal, Sanjeev (2007), "Connections: an introduction to the economics of networks," Princeton University Press.

Greenhill, Mckay, and Wang. Asymptotic enumeration of sparse 0-1 matrices with irregular row and column sums. J. Combinatorial Theory, Ser. A, 113 (2006) 291-324.

Greif, Avner (1993), "Contract Enforceability and Economic Institutions in Early Trade: The Maghribi Traders' Coalition," American Economic Review, 83, 525-548.

Hardle, Wolfgang, and Alan P. Kirman (1995), "Nonclassical Demand: A ModelFree Examination of Price-Quantity Relations in the Marseille Fish Market," Journal of Econometrics, 67, 227-257. 
Jackson, Matthew O. and Leeat Yariv (2007), "Diffusion of Behavior and Equilibrium Properties in Network Games," The American Economic Review (Papers and Proceedings), Volume 97(2), pages 92-98.

Jackson, Matthew O. (2008), "Social and Economic Networks," Princeton University Press.

Jackson, Matthew O., Tomas Rodriguez-Barraquer, and Xu Tan (2010), "Social Capital and Social Quilts: Network Patterns of Favor Exchange," working paper.

Kandori, Michihiro (1992), "Social Norms and Community Enforcement," Review of Economic Studies, 59, 63-80.

Kanoria, Yashodhan, Mohsen Bayati, Christian Borgs, Jennifer Chayes, and Andrea Montanari (2010), "Fast Convergence of Natural Bargaining Dynamics in Exchange Networks," forthcoming ACM-SIAM Symposium on Discrete Algorithms (SODA), 2011.

Karlan, Dean, Tanya Rosenblat, Markus M. Mobius, and Adam G. Szeidl (2009), "Trust and Social Collateral," Quarterly Journal of Economics, Vol. 124, No. 3, pp. $1307-1361$.

Kets, Willemien (2010), "Robustness of Equilibria in Anonymous Local Games," working paper.

Kinateder, Markus (2008), "Repeated Games Played in a Network," FEEM Working Paper No. 22.2008.

Kirman, Alan P., and Nicolaas J. Vriend (2000), "Learning to Be Loyal. A Study of the Marseille Fish Market", in Domenico Delli Gatti, Mauro Gallegati, and Alan P. Kirman "Interaction and Market Structure: Essays on Heterogeneity in Economics," Springer.

Kranton, Rachel and Deborah Minehart (2001), "A Theory of Buyer-Seller Networks," American Economic Review, 91, 485-508.

Krause, Manfred. A simple proof of the Gale-Ryser Theorem. The American Mathematical Monthly Vol. 103, No. 4 (Apr., 1996), pp. 335-337.

Lauer, Joseph and Nicholas C. Wormald (2007), "Large Independent Sets in Regular Graphs of Large Girth," Journal of Combinatorial Theory, Series B, 97, pp. 999-1009.

Lippert, Steffen and Giancarlo Spagnolo (2006), "Networks of Relations and Social Capital," SSE/EFI Economics and Finance Working Paper No. 570.

Macaulay, Stewart (1963), "Non-Contractual Relations in Business: A Preliminary Study," American Sociological Review, Vol. 28, No. 1, pp. 55-67.

Mailath, George J. and Larry Samuelson (2006), "Repeated games and reputations : long-run relationships," Oxford University Press, New York, NY.

McMillan, John and Christopher Woodruff (1999), "Interfirm Relationships and Informal Credit in Vietnam" Quarterly Journal of Economics, 114, 1285-1320. 
Mihm, Maximilian, Russell Toth, and Corey Lang (2009), "What Goes Around Comes Around: A theory of indirect reciprocity in networks," Cornell Center for Analytical Economics working paper \#09-07.

Miller, David A. and Ali S. Nageeb (2009), "Enforcing Cooperation in Networked Societies," in progress.

Nava, Francesco and Michele Piccione (2010), "Repeated Networked Games" in progress.

Niehaus, Paul (2010), "Filtered Social Learning, with an Application to Productivity Growth," working paper.

Resnick, Paul, Richard Zeckhauser, Eric Friedman, and Ko Kuwabara (2000), "Reputation Systems," Communications of the ACM, 43, 45-48.

Resnick, Paul and Richard Zeckhauser (2002), "Trust Among Strangers in Internet Transactions: Empirical Analysis of eBay's Reputation System," in The Economics of the Internet and E-Commerce, Michael R. Baye, editor, Volume 11 of Advances in Applied Microeconomics, Amsterdam, Elsevier Science, 127-157.

Richardson, Tom, and Ruediger Urbanke (2008), "Modern Coding Theory," Cambridge University Press.

Weber, Theophane (2010), "Correlation Decay and Decentralized Optimization in Graphical Models," Ph. D. dissertation, Massachusetts Institute of Technology. URI: http://hdl.handle.net/1721.1/58079.

Weisbuch Gerard, Alan P. Kirman, and Dorothea Herreiner (1996), "Market Organisation and Trading Relationships", The Economic Journal, 110, 411 - 436.

Wormald, Nicholas C. (1999), "Models of random regular graphs," in Surveys in Combinatorics, J.D. Lamb and D.A. Preece eds. London Mathematical Society Lecture Note Series, vol 276, pp. 239-298. Cambridge University Press, Cambridge. 